\title{
HOMOGENIZATION AND LOCALIZATION IN LOCALLY PERIODIC TRANSPORT
}

\author{
Grégoire Allaire $^{1}$, Guillaume Bal ${ }^{2}$ and Vincent Siess $^{3}$
}

\begin{abstract}
In this paper, we study the homogenization and localization of a spectral transport equation posed in a locally periodic heterogeneous domain. This equation models the equilibrium of particles interacting with an underlying medium in the presence of a creation mechanism such as, for instance, neutrons in nuclear reactors. The physical coefficients of the domain are $\varepsilon$-periodic functions modulated by a macroscopic variable, where $\varepsilon$ is a small parameter. The mean free path of the particles is also of order $\varepsilon$. We assume that the leading eigenvalue of the periodicity cell problem admits a unique minimum in the domain at a point $x_{0}$ where its Hessian matrix is positive definite. This assumption yields a concentration phenomenon around $x_{0}$, as $\varepsilon$ goes to 0 , at a new scale of the order of $\sqrt{\varepsilon}$ which is superimposed with the usual $\varepsilon$ oscillations of the homogenized limit. More precisely, we prove that the particle density is asymptotically the product of two terms. The first one is the leading eigenvector of a cell transport equation with periodic boundary conditions. The second term is the first eigenvector of a homogenized diffusion equation in the whole space with quadratic potential, rescaled by a factor $\sqrt{\varepsilon}$, i.e., of the form $\exp \left(-\frac{1}{2 \varepsilon} M\left(x-x_{0}\right) \cdot\left(x-x_{0}\right)\right)$, where $M$ is a positive definite matrix. Furthermore, the eigenvalue corresponding to this second term gives a first-order correction to the eigenvalue of the heterogeneous spectral transport problem.
\end{abstract}

Mathematics Subject Classification. 35B27, 82D75.

Received December 14, 2001.

\section{INTRODUCTION}

This paper is devoted to the homogenization of a transport equation in a locally periodic medium. We consider the following eigenvalue problem for the transport equation

$$
\left\{\begin{aligned}
\varepsilon v \cdot \nabla \phi^{\varepsilon}(x, v)+\Sigma^{\varepsilon}(x, v) \phi^{\varepsilon}(x, v)- & \int_{V} \sigma^{\varepsilon}\left(x, v^{\prime}, v\right) \phi^{\varepsilon}\left(x, v^{\prime}\right) \mathrm{d} v^{\prime} \\
& =\lambda^{\varepsilon} \int_{V} f^{\varepsilon}\left(x, v^{\prime}, v\right) \phi^{\varepsilon}\left(x, v^{\prime}\right) \mathrm{d} v^{\prime} \text { in } \Omega \times V \\
\phi^{\varepsilon}=0 \quad \text { on } \Gamma_{-}=\{(x, v) \in \partial \Omega & \times V, v \cdot n(x)<0\}
\end{aligned}\right.
$$

Keywords and phrases: Homogenization, localization, transport.

1 Centre de Mathématiques Appliquées, École Polytechnique, 91128 Palaiseau Cedex, France, and CEA Saclay, DEN/DM2S, 91191 Gif-sur-Yvette, France; e-mail: gregoire.allaire@polytechnique.fr

2 Department of Applied Physics and Applied Mathematics, Columbia University, New York, NY 10027, USA;

e-mail: gb2030@columbia.edu

3 CEA Saclay, DEN/DM2S, 91191 Gif-sur-Yvette, France; e-mail: siess@soleil.serma.cea.fr 
where $\Omega$ is a bounded convex domain, $V$ is the velocity space, and the coefficients (or cross-sections) are periodically oscillating functions defined by

$$
\Sigma^{\varepsilon}(x, v)=\Sigma\left(x, \frac{x}{\varepsilon}, v\right), \sigma^{\varepsilon}\left(x, v^{\prime}, v\right)=\sigma\left(x, \frac{x}{\varepsilon}, v^{\prime}, v\right), f^{\varepsilon}\left(x, v^{\prime}, v\right)=f\left(x, \frac{x}{\varepsilon}, v^{\prime}, v\right) .
$$

The $\varepsilon$-scaling in front of the advection term in (1) means that the mean free path is of the same order as the period $\varepsilon$. In nuclear reactor physics, equation (1) is known as the criticality problem for neutron transport. It expresses the balance between the production of neutrons by fission (the right hand side of (1)) and its absorption or scattering in the reactor core and leakage at the boundary (the left hand side of (1)). The unknowns are the neutron density (or flux) $\phi^{\varepsilon}(x, v)$ and the eigenvalue $\lambda^{\varepsilon}$ (the inverse of which is called multiplication factor), which measures the balance between the production and removal of neutrons. Since only positive densities have a physical meaning, the only relevant solution turns out to be the first eigenvector (positive and unique up to a multiplicative constant). There are of course other physical motivations for the study of (1), including photon transport, radiative transfer, and semi-conductors.

Since the pioneering work of Larsen [19-21] (not to mention the previous physics literature), many papers have been devoted to the time evolution version of problem (1) (see e.g. $[8,12,16,17,27]$ ). The eigenvalue problem (1) was studied in $[2,7]$. In all these papers, there is always an assumption of pure periodicity, which means that the coefficients in (2) depend solely on the fast variable $y=x / \varepsilon$ and not on the slow variable $x$. In truth the papers [17] and [16] do not make precisely such an assumption but rather assume that the resulting local behavior is not oscillating, i.e. depends on $x$ but not on $y=x / \varepsilon$. In any case, the possibility of a strong coupling of the fast and slow variables has never been explored in full generality with coefficients defined by (2). We address this problem under a suitable structural assumption and show that the homogenized limit is very different from that obtained in the purely periodic case. To explain our results we introduce the cell eigenvalue problem which is defined for each $x \in \Omega$ by

$$
\left\{\begin{array}{l}
v \cdot \nabla_{y} \psi+\Sigma \psi=\int_{V} \sigma \psi \mathrm{d} v^{\prime}+\lambda^{\infty}(x) \int_{V} f \psi \mathrm{d} v^{\prime} \text { in } Y \\
y \mapsto \psi(x, y, v) Y \text { - periodic, }
\end{array}\right.
$$

where $\left(\lambda^{\infty}(x), \psi(x, y, v)\right)$ is the first or leading eigencouple and $Y=(0,1)^{N}$. Our structural assumption is that the function $x \mapsto \lambda^{\infty}(x)$ admits a unique minimum at $x_{0} \in \Omega$ and that its Hessian matrix is positive definite at $x_{0}$. We also make a no-drift assumption which amounts to a phase-space symmetry condition (this assumption can be removed as was shown in [7]). Our main result (see (32) for a formal asymptotic result and Th. 3.2 for a precise statement) is that, asymptotically as $\varepsilon$ tends to zero, the first eigenfunction of (1) behaves as

$$
\phi^{\varepsilon}(x, v) \approx \psi\left(x_{0}, \frac{x}{\varepsilon}, v\right) \exp \left(-\frac{M\left(x-x_{0}\right) \cdot\left(x-x_{0}\right)}{2 \varepsilon}\right),
$$

where $M$ is a positive definite matrix depending on some homogenized properties and on the Hessian of $\lambda^{\infty}$ at $x_{0}$. It is clear from (4) that $\phi^{\varepsilon}$ is localized near $x_{0}$ at a length scale of order $\sqrt{\varepsilon}$. Furthermore, the first eigenvalue can be expanded as

$$
\lambda^{\varepsilon}=\lambda^{\infty}\left(x_{0}\right)+\varepsilon \lambda_{1}+o(\varepsilon)
$$

where $\lambda_{1}$ is explicit in terms of $M$ and other homogenized quantities (see $(26,31)$, and Rem. 3.1). Even when the coefficients do not oscillate (i.e. are functions of $x$ but not of $x / \varepsilon$ ) the asymptotic result (4) is non trivial and new to the best of our knowledge. Our results extend a previous study made in [4] on a similar eigenvalue problem for a diffusion equation. Related results on diffusion equations may be found in $[3,18]$, and $[24]$. 
The paper is organized as follows. In the next section we introduce our notation and detailed assumptions, and we recall some basic mathematical properties of transport equations. Section 2 is devoted to the homogenization of (1) by means of asymptotic expansions. This method is formal but it has the advantage of being easily accessible without any knowledge of functional analysis. Section 3 is devoted to a detailed presentation of the rigorous convergence results concerning the homogenization of (1). The proofs of these results are given in Sections 4 and 5. More precisely, Section 4 focuses on a priori estimates for a source problem associated with (1), while Section 5 is concerned with the proof of the homogenization process, using the two-scale convergence method. Finally, Section 6 is devoted to some auxiliary cell problems.

\section{Assumptions and notation}

This section is devoted to a precise statement of our main assumptions and to a brief presentation of our notation and of classical results in transport theory that are necessary for our analysis. We include these known results (without proofs) for completeness, and we refer to, e.g. $[2,6,11]$ for details. We first give the detailed assumptions on the physical parameters that are used throughout this paper.

(H1) The domain $\Omega$ is a convex bounded open set of $\mathbb{R}^{N}$, and the velocity space $V$ is a compact subset of $\mathbb{R}^{N}$ which does not contain 0 . Furthermore $V$ is assumed to be the closure of an open set, and its $N$-dimensional measure is normalized to have $|V|=1$.

(H2) The cross-sections $\Sigma(x, y, v), \sigma\left(x, y, v^{\prime}, v\right)$, and $f\left(x, y, v^{\prime}, v\right)$ are of class $\mathcal{C}^{2}$ in $x \in \bar{\Omega}$ and measurable in $y$. They are positive, bounded $Y$-periodic functions in $y$, where $Y=(0,1)^{N}$ is the unit cube, and there exists a positive constant $C>0$ such that, for a.e. $\left(x, y, v, v^{\prime}\right)$,

$$
\begin{aligned}
f\left(x, y, v^{\prime}, v\right) & \geq C, \\
\Sigma(x, y, v)-\int_{Y} \sigma\left(x, y, v^{\prime}, v\right) \mathrm{d} v^{\prime} & \geq C, \\
\Sigma(x, y, v)-\int_{V} \sigma\left(x, y, v, v^{\prime}\right) \mathrm{d} v^{\prime} & \geq C .
\end{aligned}
$$

Remark 1.1. There are possible variants of assumption (H2) which may be more appropriate for some applications. For example, as it stands, (H2) implies that particle creation occurs everywhere, which is not the case in neutron transport where fission takes place only in the nuclear fuel and not in the moderator. This can easily be corrected by replacing the first inequality in (5) by

$$
\sigma\left(x, y, v^{\prime}, v\right)+f\left(x, y, v^{\prime}, v\right) \geq C, \quad \text { a.e. }\left(x, y, v, v^{\prime}\right)
$$

with $f \geq 0$ and $f \not \equiv 0$. This implies that the sum of fission and scattering is positive everywhere. Up to some additional technicalities, all our results also hold in this framework.

Introducing the Hilbert space

$$
W^{2}(\Omega \times V)=\left\{u \in L^{2}(\Omega \times V), v \cdot \nabla u \in L^{2}(\Omega \times V)\right\},
$$

assumptions (H1) and (H2) allow to state the following existence result.

Theorem 1.2. The spectral problem (1) has at most a countable number of eigenvalues and of associated eigenvectors in $W^{2}(\Omega \times V)$. Furthermore, there exists a real and positive eigenvalue, of smallest modulus, with multiplicity one, and such that its associated eigenvector is the unique (up to a multiplicative constant) positive eigenvector of (1).

The proof of Theorem 1.2, which is in the spirit of other results in [11] (Chap. 21), can be found in [6]. As a consequence of Theorem 1.2, only the first eigenvector of (1) has a physical meaning as a particle density. 
As we shall see in the sequel, the asymptotic behavior of the eigenvectors of (1) is partly governed by the first eigenvector of another eigenvalue problem, similar to (1) but posed in the unit periodicity cell $Y$ with periodic boundary conditions. Denoting by $\lambda^{\infty}(x)$ and $\psi(x, y, v)$ its first eigenvalue and eigenvector, the infinite medium problem is defined for each parameter $x \in \Omega$ by

$$
\left\{\begin{aligned}
v \cdot \nabla_{y} \psi(x, y, v)+\Sigma(x, y, v) \psi(x, y, v)= & \int_{V} \sigma\left(x, y, v^{\prime}, v\right) \psi\left(x, y, v^{\prime}\right) \mathrm{d} v^{\prime} \\
& +\lambda^{\infty}(x) \int_{V} f\left(x, y, v^{\prime}, v\right) \psi\left(x, y, v^{\prime}\right) \mathrm{d} v^{\prime} \\
y \mapsto \psi(x, y, v) Y \text { - periodic. } &
\end{aligned}\right.
$$

We shall also need an adjoint problem to (7), which has the same first eigenvalue $\lambda^{\infty}(x)$ with a different first eigenvector $\psi^{*}(x, y, v)$. Introducing the adjoint cross-sections

$$
f^{*}\left(x, y, v^{\prime}, v\right)=f\left(x, y, v, v^{\prime}\right) \text { and } \sigma^{*}\left(x, y, v^{\prime}, v\right)=\sigma\left(x, y, v, v^{\prime}\right)
$$

this adjoint problem is defined by

$$
\left\{\begin{aligned}
-v \cdot \nabla_{y} \psi^{*}(x, y, v)+\Sigma(x, y, v) \psi^{*}(x, y, v)= & \int_{V} \sigma^{*}\left(x, y, v^{\prime}, v\right) \psi^{*}\left(x, y, v^{\prime}\right) \mathrm{d} v^{\prime} \\
& +\lambda^{\infty}(x) \int_{V} f^{*}\left(x, y, v^{\prime}, v\right) \psi^{*}\left(x, y, v^{\prime}\right) \mathrm{d} v^{\prime} \\
y \mapsto \psi^{*}(x, y, v) Y \text { - periodic. } &
\end{aligned}\right.
$$

As a corollary of Theorem 1.2 there exist leading eigenvalues and eigenvectors for the cell problems (7) and (8), which can be chosen positive.

Theorem 1.3. There exists a common eigenvalue $\lambda^{\infty}(x)$ to both problems (7) and (8), which is real, positive, of smallest modulus, with multiplicity one, and such that the respective eigenvectors $\psi$ and $\psi^{*}$ are positive elements of $W^{2}(Y \times V)$.

We are now in a position to give our next assumptions.

(H3) We assume that $x \mapsto \lambda^{\infty}(x)$ admits a unique minimum at $x_{0} \in \Omega$ and that its Hessian matrix is positive definite at $x_{0}$. Without loss of generality, we suppose that $x_{0}=0$

$$
\lambda^{\infty}(x)=\lambda^{\infty}(0)+x_{k} x_{l} \lambda_{k l}^{2}+o\left(|x|^{2}\right),
$$

and $\left(\lambda_{k l}^{2}\right)_{1 \leq k, l \leq N}$ is a positive definite matrix.

(H4) Finally, we need the additional hypothesis that the drift flux

$$
J(x)=\int_{Y} \int_{V} v \psi(x, y, v) \psi^{*}(x, y, v) \mathrm{d} y \mathrm{~d} v
$$

vanishes at $x=0$, i.e. $J(0)=0$.

Remark 1.4. Assumption (H3) is somehow generic as soon as we are interested in non-constant eigenvalues $\lambda^{\infty}(x)$. Let us mention at least one (simple) case when it holds true: take $\Sigma(x, y, v)=\Sigma^{0}(y, v), \sigma\left(x, y, v^{\prime}, v\right)$ $=\sigma^{0}\left(y, v^{\prime}, v\right)$ and $f\left(x, y, v^{\prime}, v\right)=k(x) f^{0}\left(y, v^{\prime}, v\right)$ so that $\lambda^{\infty}(x)=\lambda^{0} / k(x)$, and (H3) is satisfied for a properly chosen function $k(x)$.

Remark 1.5. The assumption $(\mathrm{H} 4), J(0)=0$, can be interpreted as a symmetry condition in the phase space (or a no-drift condition), as explained in [2] or [6]. It is quite usual in this type of problem (for example, it is 
imposed in $[16,17])$. In most practical cases, assumption (H4) holds true. For example, $J(0)=0$ when $V=-V$ (in the sense that $v \in V \Rightarrow-v \in V$ ) and the cross sections do not depend on the velocity variable (this is the so-called one-velocity isotropic case), or when the cross-sections are symmetric with respect to $v$, and the cell $Y$ has cubic symmetry. The paper [7] addresses the case when the drift $J(0)$ is not zero and the coefficients are purely periodic functions. We briefly discuss another possible hypothesis when $J(0) \neq 0$ in Section 6 .

In the sequel we shall also need the following results. Since the smallest eigenvalue $\lambda^{\infty}(x)$ is simple, the classical Fredholm alternative for compact operators yields an existence result for (7) with a source term.

Proposition 1.6. Let $x \in \Omega$ be fixed and let $\lambda^{\infty}(x)$ and $\psi(x, y, v)$ be the first eigenvalue and eigenvector of (7). Let $S(x, y, v)$ be a source term in $L^{2}(Y \times V)$. Then there exists a solution $\varphi(x, y, v) \in W^{2}(Y \times V)$ of

$$
\left\{\begin{aligned}
v \cdot \nabla_{y} \varphi(x, y, v)+\Sigma(x, y, v) \varphi(x, y, v)= & \int_{V} \sigma\left(x, y, v^{\prime}, v\right) \varphi\left(x, y, v^{\prime}\right) \mathrm{d} v^{\prime} \\
& +\lambda^{\infty}(x) \int_{V} f\left(x, y, v^{\prime}, v\right) \varphi\left(x, y, v^{\prime}\right) \mathrm{d} v^{\prime}+S(x, y, v) \\
y \mapsto \varphi(x, y, v) Y-\text { periodic } &
\end{aligned}\right.
$$

if and only if $S$ is orthogonal to the first eigenvector $\psi^{*}$ of (8), i.e., $S$ satisfies the compatibility condition

$$
\int_{Y} \int_{V} S(x, y, v) \psi^{*}(x, y, v) \mathrm{d} y \mathrm{~d} v=0 .
$$

Furthermore, if it exists, the solution $\varphi$ is unique up to the addition of a multiple of $\psi$.

The first eigenvectors $\psi$ and $\psi^{*}$ are bounded from above and below by positive constants as stated in the following proposition, based on the averaging lemma [14] and Sobolev inequalities, the proof of which can be found in [2].

Proposition 1.7. Let $\psi$ and $\psi^{*}$ be the first positive eigenvectors of problems (7) and (8), respectively. Then there exist two positive constants $0<C \leq C^{\prime}$ such that, for a.e. $(x, y, v)$,

$$
0<C \leq \psi(x, y, v) \leq C^{\prime} \quad \text { and } \quad 0<C \leq \psi^{*}(x, y, v) \leq C^{\prime} .
$$

Finally, we state a compactness result for transport equations which is a straightforward variation of the classical velocity averaging lemma of $[14,15]$.

Lemma 1.8. Let $u^{\varepsilon}(z, v)$ be a family of functions of $W^{2}\left(\mathbb{R}^{N} \times V\right)$ such that there exists a positive constant $C$ independent of $\varepsilon$ satisfying

$$
\left\|(1+|z|) u^{\varepsilon}(z, v)\right\|_{L^{2}\left(\mathbb{R}^{N} \times V\right)}+\left\|v \cdot \nabla u^{\varepsilon}(z, v)\right\|_{L^{2}\left(\mathbb{R}^{N} \times V\right)} \leq C .
$$

Then the family $\int_{V} u^{\varepsilon}(z, v) \mathrm{d} v$ is relatively compact in $L^{2}\left(\mathbb{R}^{N}\right)$.

In the sequel, we always assume that hypotheses (H1-H4) hold.

\section{AsYmptotic EXPANSION}

To address the phenomenon of concentration and homogenization for (1) the simplest approach is the classical technique of two-scale asymptotic expansions, coupled with Taylor expansions around the concentration point $x=0$. This is a formal method which has the advantage of avoiding all the fine points of functional analysis that are required for a convergence proof. Therefore, we believe it is interesting even though we shall not use the results of this section in our convergence theorem of Section 3. Remark that it is possible to justify the 
asymptotic expansion by a careful study of the remainder terms, but this method has two drawbacks. First, it requires smoother physical data. Second, it gives a full justification only of the first term in the expansion although the expansion contains four terms. This phenomenon is well documented in [4].

The first step of the derivation is to approximate the following functions around $x=0$ by their Taylor expansions (the Einstein convention of summation over repeated indices is used)

$$
\left\{\begin{aligned}
\Sigma(x, y, v) & =\Sigma^{0}(y, v)+x_{k} \Sigma_{k}^{1}(y, v)+x_{k} x_{l} \Sigma_{k l}^{2}(y, v)+o\left(|x|^{2}\right) \\
\sigma\left(x, y, v^{\prime}, v\right) & =\sigma^{0}\left(y, v^{\prime}, v\right)+x_{k} \sigma_{k}^{1}\left(y, v^{\prime}, v\right)+x_{k} x_{l} \sigma_{k l}^{2}\left(y, v^{\prime}, v\right)+o\left(|x|^{2}\right) \\
f\left(x, y, v^{\prime}, v\right) & =f^{0}\left(y, v^{\prime}, v\right)+x_{k} f_{k}^{1}\left(y, v^{\prime}, v\right)+x_{k} x_{l} f_{k l}^{2}\left(y, v^{\prime}, v\right)+o\left(|x|^{2}\right) \\
\psi(x, y, v) & =\psi^{0}(y, v)+x_{k} \psi_{k}^{1}(y, v)+x_{k} x_{l} \psi_{k l}^{2}(y, v)+o\left(|x|^{2}\right) \\
\lambda^{\infty}(x) & =\lambda^{\infty}(0)+x_{k} x_{l} \lambda_{k l}^{2}+o\left(|x|^{2}\right)
\end{aligned}\right.
$$

Here we use the following notation. For any function $g(x)$, we define $g_{k}^{1}=\frac{\partial g}{\partial x_{k}}(x=0)$, and $g_{k l}^{2}=\frac{1}{2} \frac{\partial^{2} g}{\partial x_{k} \partial x_{l}}(x=0)$. We also define $2\left(\lambda_{k l}^{2}\right)$ as the Hessian matrix of $x \mapsto \lambda^{\infty}(x)$ at $x=0$, where $\lambda^{\infty}$ is assumed to reach its minimum.

Following [4], where a similar problem for the diffusion equation is considered, we introduce the following ansatz for the first eigenpair of (1)

$$
\left\{\begin{aligned}
\phi^{\varepsilon}(x, v)= & \exp \left(-\frac{M x \cdot x}{2 \varepsilon}\right)\left[\phi^{0}\left(\frac{x}{\varepsilon}, v\right)+x_{k} \phi_{k}^{1}\left(\frac{x}{\varepsilon}, v\right)+x_{k} x_{l} \phi_{k l}^{2}\left(\frac{x}{\varepsilon}, v\right)\right. \\
& \left.+\varepsilon \phi^{3}\left(\frac{x}{\varepsilon}, v\right)+r^{\varepsilon}(x, v)\right] \\
\lambda^{\varepsilon}= & \lambda^{0}+\varepsilon \lambda^{1}+o(\varepsilon)
\end{aligned}\right.
$$

where $\phi^{0}, \phi_{k}^{1}, \phi_{k l}^{2}$, and $\phi^{3}$ are $Y$-periodic functions in their first argument to be determined, $M$ is an unknown symmetric positive definite matrix, and $r^{\varepsilon}$ is a small remainder term. The matrix $M$ being positive definite, we notice that, for any $p \in \mathbb{N}$,

$$
\frac{\left\|x^{p} \exp \left(-\frac{M x \cdot x}{2 \varepsilon}\right)\right\|_{L^{r}(\Omega)}}{\left\|\exp \left(-\frac{M x \cdot x}{2 \varepsilon}\right)\right\|_{L^{r}(\Omega)}} \approx O\left(\varepsilon^{\frac{p}{2}}\right) \text { for any } r \in[1,+\infty] .
$$

Assuming that the first term $\phi^{0}$ in the asymptotic expansion is normalized such that its $L^{2}$-norm is 1 , then the second term $\phi^{1}=\left(\phi_{k}^{1}\right)$ will be of order $\sqrt{\varepsilon}$, and the third and fourth terms $\phi^{2}=\left(\phi_{k l}^{2}\right)$ and $\phi^{3}$ of order $\varepsilon$. After some algebra, we find

$$
\begin{aligned}
\nabla\left(\phi^{\varepsilon}\right)= & \frac{1}{\varepsilon} \exp \left(-\frac{M x \cdot x}{2 \varepsilon}\right)\left[\nabla_{y} \phi^{0}\left(\frac{x}{\varepsilon}, v\right)+\left(x_{k} \nabla_{y} \phi_{k}^{1}-M x \phi^{0}\right)\left(\frac{x}{\varepsilon}, v\right)\right. \\
& \left.+\left(x_{k} x_{l} \nabla_{y} \phi_{k l}^{2}-M x x_{k} \phi_{k}^{1}\right)\left(\frac{x}{\varepsilon}, v\right)+\varepsilon\left(\phi^{1}+\nabla_{y} \phi^{3}\right)\left(\frac{x}{\varepsilon}, v\right)+r^{\prime} \varepsilon(x, v)\right]
\end{aligned}
$$

where, as usual, $\nabla_{x}$ and $\nabla_{y}$ denote partial derivatives with respect to the slow variable $x$ and fast variable $y$, respectively, and $r^{\prime} \varepsilon$ is a remainder term. Identifying all terms according to their power in $x$ and $\varepsilon$, we obtain a cascade of equations from which we keep the four first ones. The zeroth order terms yield

$$
v \cdot \nabla_{y} \phi^{0}+\Sigma^{0} \phi^{0}=\int_{V} \sigma^{0} \phi^{0}+\lambda^{0} \int_{V} f^{0} \phi^{0}
$$

The first order terms in $x$ give for all $k \in\{1, \ldots, N\}$

$$
v_{i}\left(\partial_{y_{i}} \phi_{k}^{1}-M_{i k} \phi^{0}\right)+\left(\Sigma^{0} \phi_{k}^{1}+\Sigma_{k}^{1} \phi^{0}\right)=\int_{V}\left(\sigma^{0} \phi_{k}^{1}+\sigma_{k}^{1} \phi^{0}\right)+\lambda^{0} \int_{V}\left(f^{0} \phi_{k}^{1}+f_{k}^{1} \phi^{0}\right),
$$


where $\partial_{y_{i}}$ denotes the $i$-th component of the gradient $\nabla_{y}$. The second order terms in $x$ give for all $k, l$ $\in\{1, \ldots, N\}$,

$$
v_{i}\left(\partial_{y_{i}} \phi_{k l}^{2}-M_{i l} \phi_{k}^{1}\right)+\left(\Sigma^{0} \phi_{k l}^{2}+\Sigma_{k}^{1} \phi_{l}^{1}+\Sigma_{k l}^{2} \phi^{0}\right)=\int_{V}\left(\sigma^{0} \phi_{k l}^{2}+\sigma_{k}^{1} \phi_{l}^{1}+\sigma_{k l}^{2} \phi^{0}\right)+\lambda^{0} \int_{V}\left(f^{0} \phi_{k l}^{2}+f_{k}^{1} \phi_{l}^{1}+f_{k l}^{2} \phi^{0}\right)
$$

(the formula (14) has to be symmetrized with respect to $k, l$ since $x_{k} x_{l}$ is itself symmetric), and the first order terms in $\varepsilon$ yield

$$
v_{i}\left(\phi_{i}^{1}+\partial_{y_{i}} \phi^{3}\right)+\Sigma^{0} \phi^{3}=\int_{V} \sigma^{0} \phi^{3}+\lambda^{0} \int_{V} f^{0} \phi^{3}+\lambda^{1} \int_{V} f^{0} \phi^{0} .
$$

Eventually, solving these equations leads to the asymptotic behavior of $\phi^{\varepsilon}$. Equation (12) allows us to determine $\phi^{0}$ and $\lambda^{0}$. Equations (13) and (14) allow us to determine $\phi_{k}^{1}$ and $\phi_{k l}^{2}$, and some compatibility conditions will give us the expression for $M$. Finally, equation (15) determines the $\varepsilon$ order term $\phi^{3}$ and its solvability condition gives the first order corrector $\lambda^{1}$ for the eigenvalue.

\subsection{Zeroth order equation}

Since at $x=0$ the functions in (10) coincide with the zero-order terms in their Taylor expansions, the zeroth order equation (12) is simply the periodic cell problem (7) at $x=0$. Thanks to Theorem 1.3, equation (12) has thus a unique positive solution given by

$$
\phi^{0}(y, v)=\psi^{0}(y, v), \quad \lambda^{0}=\lambda^{\infty}(0)
$$

where $\psi^{0}(y, v)$ is equal to $\psi(x=0, y, v)$ up to some multiplicative constant depending on the normalization of $\phi^{\varepsilon}$.

\subsection{First order equation in $x$}

For each $k$, equation (13) can be written as follows

$$
v \cdot \nabla_{y} \phi_{k}^{1}+\Sigma^{0} \phi_{k}^{1}=\int_{V} \sigma^{0} \phi_{k}^{1}+\lambda^{0} \int_{V} f^{0} \phi_{k}^{1}+g_{k}^{1},
$$

where the source term $g_{k}^{1}$ is given by

$$
g_{k}^{1}=v_{i} M_{i k} \psi^{0}-\Sigma_{k}^{1} \psi^{0}+\int_{V} \sigma_{k}^{1} \psi^{0}+\lambda^{0} \int_{V} f_{k}^{1} \psi^{0} .
$$

According to the Fredholm alternative of Proposition 1.6, these equations can be solved if and only if the source-term $g_{k}^{1}$ is orthogonal to $\psi^{0 *}(y, v)=\psi^{*}(0, y, v)$, i.e.,

$$
\int_{Y} \int_{V}\left[v_{i} M_{i k} \psi^{0}-\Sigma_{k}^{1} \psi^{0}+\left(\int_{V} \sigma_{k}^{1} \psi^{0}\right)+\lambda^{0}\left(\int_{V} f_{k}^{1} \psi^{0}\right)\right] \psi^{0 *}=0 .
$$

Upon differentiating the infinite medium equation (7) with respect to $x$ at $x=0$, we obtain

$$
v \cdot \nabla_{y} \psi_{k}^{1}+\Sigma^{0} \psi_{k}^{1}+\Sigma_{k}^{1} \psi^{0}=\int_{V} \sigma^{0} \psi_{k}^{1}+\int_{V} \sigma_{k}^{1} \psi^{0}+\lambda^{0} \int_{V} f^{0} \psi_{k}^{1}+\lambda^{0} \int_{V} f_{k}^{1} \psi^{0}
$$


which admits $\psi_{k}^{1}=\left(\partial_{x_{k}} \psi^{0}\right)(x=0)$ as a solution. Still, it admits a solvability condition given by

$$
\int_{Y} \int_{V}\left[-\Sigma_{k}^{1} \psi^{0}+\left(\int_{V} \sigma_{k}^{1} \psi^{0}\right)+\lambda^{0}\left(\int_{V} f_{k}^{1} \psi^{0}\right)\right] \psi^{0 *}=0
$$

Thus, equation (13) is solvable if and only if

$$
\int_{Y} \int_{V} v_{i} M_{i k} \psi^{0} \psi^{0 *}=0
$$

or equivalently if and only if

$$
M \int_{Y} \int_{V} v \psi^{0} \psi^{0 *}=0
$$

The latter equation holds thanks to hypothesis (H4), hence (13) admits solutions. We shall see later on that $M$ is a symmetric positive definite matrix, so (H4) is a necessary and sufficient solvability condition for equation (13). The solution $\phi_{k}^{1}$ of (17) can be written as the sum of two terms

$$
\phi_{k}^{1}=-M_{k j} \chi^{j}+\psi_{k}^{1}
$$

where $\psi_{k}^{1}$ is defined in (10) and $\chi^{j}$ is the solution of the following equation

$$
v \cdot \nabla_{y} \chi^{j}+\Sigma^{0} \chi^{j}=\int_{V} \sigma^{0} \chi^{j}+\lambda^{0} \int_{V} f^{0} \chi^{j}-v_{j} \psi^{0} .
$$

Since $J(0)=0$, the solvability condition of this equation is verified, and therefore $\chi^{j}$ is uniquely defined up to a multiple of $\psi^{0}$. Notice that terms proportional to $\psi^{0}$ can be incorporated into $\phi^{0}$ in (16).

\subsection{Second order equation in $x$}

For each $k, l$, equation (14) can be rewritten as

$$
v \cdot \nabla_{y} \phi_{k l}^{2}+\Sigma^{0} \phi_{k l}^{2}=\int_{V} \sigma^{0} \phi_{k l}^{2}+\lambda^{0} \int_{V} f^{0} \phi_{k l}^{2}+g_{k l}^{2},
$$

where the source term is given by

$$
g_{k l}^{2}=v_{i} M_{i l} \phi_{k}^{1}-\Sigma_{k}^{1} \phi_{l}^{1}-\Sigma_{k l}^{2} \psi^{0}+\int_{V} \sigma_{k}^{1} \phi_{l}^{1}+\int_{V} \sigma_{k l}^{2} \psi^{0}+\lambda^{0} \int_{V} f_{k}^{1} \phi_{l}^{1}+\lambda^{0} \int_{V} f_{k l}^{2} \psi^{0} .
$$

In truth, $g_{k l}^{2}$ is symmetric with respect to $k, l$ so that equation (23) should be symmetrized (for brevity we do not include the symmetric terms in (23)). Again, this equation admits a solution if and only if $g_{k l}^{2}$ is orthogonal to $\psi^{0 *}$. Owing to $(21)$, the source term $g_{k l}^{2}$ can be recast as

$$
\begin{aligned}
g_{k l}^{2}= & -v_{i} M_{i l} M_{k j} \chi^{j}+v_{i} M_{i l} \psi_{k}^{1}+\Sigma_{k}^{1} M_{l j} \chi^{j}-\Sigma_{k}^{1} \psi_{l}^{1}-\Sigma_{k l}^{2} \psi^{0} \\
& +\int_{V}\left(-\sigma_{k}^{1} M_{l j} \chi^{j}+\sigma_{k}^{1} \psi_{l}^{1}+\sigma_{k l}^{2} \psi^{0}\right)+\lambda^{0} \int_{V}\left(-f_{k}^{1} M_{l j} \chi^{j}+f_{k}^{1} \psi_{l}^{1}+f_{k l}^{2} \psi^{0}\right),
\end{aligned}
$$


which after reordering yields

$$
\begin{aligned}
g_{k l}^{2}= & -M_{i l} M_{k j} v_{i} \chi^{j}+M_{i l}\left(v_{i} \psi_{k}^{1}+\Sigma_{k}^{1} \chi^{i}-\int_{V} \sigma_{k}^{1} \chi^{i}-\lambda^{0} \int_{V} f_{k}^{1} \chi^{i}\right) \\
& +\left(-\Sigma_{k}^{1} \psi_{l}^{1}-\Sigma_{k l}^{2} \psi^{0}+\int_{V}\left(\sigma_{k}^{1} \psi_{l}^{1}+\sigma_{k l}^{2} \psi^{0}\right)+\lambda^{0} \int_{V}\left(f_{k}^{1} \psi_{l}^{1}+f_{k l}^{2} \psi^{0}\right)\right) .
\end{aligned}
$$

Upon differentiating the infinite medium equation (7) twice in $x$ at $x=0$, we obtain

$$
\begin{aligned}
v \cdot \nabla_{y} \psi_{k l}^{2} & +\Sigma^{0} \psi_{k l}^{2}+\Sigma_{k}^{1} \psi_{l}^{1}+\Sigma_{k l}^{2} \psi^{0}=\int_{V}\left(\sigma^{0} \psi_{k l}^{2}+\sigma_{k}^{1} \psi_{l}^{1}+\sigma_{k l}^{2} \psi^{0}\right) \\
& +\lambda^{0} \int_{V}\left(f^{0} \psi_{k l}^{2}+f_{k}^{1} \psi_{l}^{1}+\sigma_{k l}^{2} \psi^{0}\right)+\lambda_{k l}^{2} \int_{V} f^{0} \psi^{0}
\end{aligned}
$$

which admits a solution by construction. We rewrite this equation as

$$
\begin{aligned}
v \cdot \nabla_{y} \psi_{k l}^{2}+\Sigma^{0} \psi_{k l}^{2}= & \int_{V} \sigma^{0} \psi_{k l}^{2}+\lambda^{0} \int_{V} f^{0} \psi_{k l}^{2} \\
& +\left[-\Sigma_{k}^{1} \psi_{l}^{1}-\Sigma_{k l}^{2} \psi^{0}+\int_{V}\left(\sigma_{k}^{1} \psi_{l}^{1}+\sigma_{k l}^{2} \psi^{0}\right)+\lambda^{0} \int_{V}\left(f_{k}^{1} \psi_{l}^{1}+f_{k l}^{2} \psi^{0}\right)+\lambda_{k l}^{2} \int_{V} f^{0} \psi^{0}\right]
\end{aligned}
$$

Its solvability condition reads

$$
\int_{Y} \int_{V}\left[-\Sigma_{k}^{1} \psi_{l}^{1}-\Sigma_{k l}^{2} \psi^{0}+\int_{V}\left(\sigma_{k}^{1} \psi_{l}^{1}+\sigma_{k l}^{2} \psi^{0}\right) \lambda^{0} \int_{V}\left(f_{k}^{1} \psi_{l}^{1}+f_{k l}^{2} \psi^{0}\right)+\lambda_{k l}^{2} \int_{V} f^{0} \psi^{0}\right] \psi^{0 *}=0 .
$$

Thus, the solvability condition of (14) is

$$
\begin{aligned}
-M_{i l} M_{k j} \int_{Y} \int_{V} v_{i} \chi^{j} \psi^{0 *}+M_{i l} \int_{Y} \int_{V}\left[v_{i} \psi_{k}^{1}+\Sigma_{k}^{1} \chi^{i}-\left(\int_{V} \sigma_{k}^{1} \chi^{i}\right)-\lambda^{0}\left(\int_{V} f_{k}^{1} \chi^{i}\right)\right] \psi^{0 *} & -\lambda_{k l}^{2} \int_{Y} \int_{V}\left(\int_{V} f^{0} \psi^{0}\right) \psi^{0 *}=0 .
\end{aligned}
$$

As explained before, this equation has to be symmetrized with respect to $k$ and $l$. This yields a quadratic matrix equation for the unknown $M$, which reads

$$
M_{k j} \bar{D}_{j i}^{S} M_{i l}+\bar{B}_{k i} M_{i l}+M_{k i} \bar{B}_{i l}=\bar{A}_{k l}
$$

or

$$
M \bar{D}^{S} M+\bar{B} M+M \bar{B}^{*}=\bar{A}
$$

where

$$
\begin{gathered}
\bar{A}_{k l}=\lambda_{k l}^{2} \int_{Y} \int_{V} \int_{V} f^{0}\left(y, v^{\prime}, v\right) \psi^{0}(y, v) \psi^{0 *}\left(y, v^{\prime}\right) \mathrm{d} y \mathrm{~d} v \mathrm{~d} v^{\prime}, \\
\bar{B}_{k i}=\int_{Y} \int_{V}\left[v_{i} \psi_{k}^{1}+\Sigma_{k}^{1} \chi^{i}-\left(\int_{V} \sigma_{k}^{1} \chi^{i}\right)-\lambda^{0}\left(\int_{V} f_{k}^{1} \chi^{i}\right)\right] \psi^{0 *},
\end{gathered}
$$


and $\bar{D}^{S}$ denotes the symmetrical part of $\bar{D}$, which is given by the Kubo formula (see $[8,19,27]$ )

$$
\bar{D}_{i j}=-\int_{Y} \int_{V} v_{i} \chi^{j} \psi^{0 *}
$$

Equation (26) is a Riccati equation, which is classical in Control Theory. The following theorem, which can be found in [26] (pp. 225-235) or in [25], ensures that this equation admits a unique symmetric positive definite solution.

Theorem 2.1. Let $D$ and $A$ be symmetric definite positive square matrices, and let $B$ be a square matrix of the same size. Let us consider the Riccati matrix equation

$$
M D M+B M+M B^{*}=A .
$$

Then there exists a unique symmetric definite positive solution $M$ of this equation.

Remark 2.2. In order to compare the results given by asymptotic expansions and two-scale convergence, it is worth noticing that

$$
\bar{B}_{k i}=\frac{\partial J_{i}}{\partial x_{k}}(0) .
$$

This result will be proved in Section 6. In the case where $\nabla_{x} J(0)=0$, it allows us to have a simple expression for $M$. Indeed, $M$ solves a Riccati equation now of the form

$$
M \bar{D}^{S} M=\bar{A}
$$

and $M$ is therefore given by

$$
M=\bar{D}^{S-\frac{1}{2}}\left(\bar{D}^{S \frac{1}{2}} \bar{A} \bar{D}^{S \frac{1}{2}}\right)^{\frac{1}{2}} \bar{D}^{S-\frac{1}{2}}
$$

\subsection{The $\varepsilon$ order equation}

Finally, equation (15) yields the first order corrector to the eigenvalue of our initial problem (1). Equation (15) can be written as

$$
v \cdot \nabla_{y} \phi^{3}+\Sigma^{0} \phi^{3}=\int_{V} \sigma^{0} \phi^{3}+\lambda^{0} \int_{V} f^{0} \phi^{3}+\left[-v_{i} \phi_{i}^{1}+\lambda^{1} \int_{V} f^{0} \psi^{0}\right] .
$$

The Fredholm alternative shows that this equation admits a solution if and only if

$$
\int_{Y} \int_{V} v_{i} \phi_{i}^{1} \psi^{0 *}=\lambda^{1} \int_{Y} \int_{V}\left(\int_{V} f^{0} \psi^{0}\right) \psi^{0 *}
$$

which eventually gives us the following correction to the leading eigenvalue

$$
\lambda^{1}=\frac{\int_{Y} \int_{V} v_{i} \phi_{i}^{1} \psi^{0 *}}{\int_{Y} \int_{V}\left(\int_{V} f^{0} \psi^{0}\right) \psi^{0 *}} .
$$


We have seen that $\phi_{i}^{1}=M_{i j} \chi^{j}+\psi_{i}^{1}$, and therefore, as $\psi_{i}^{1}=\partial_{x_{i}} \psi(x=0)$,

$$
\lambda^{1}=\frac{-M_{i j} \int_{Y} \int_{V} v_{i} \chi^{j} \psi^{0 *}+\int_{Y} \int_{V} v_{i} \psi_{i}^{1} \psi^{0 *}}{\int_{Y} \int_{V}\left(\int_{V} f^{0} \psi^{0}\right) \psi^{0 *}}=\frac{\operatorname{Tr}(M \bar{D})+\bar{\gamma}}{\bar{\sigma}},
$$

where $\bar{\sigma}$ and $\bar{\gamma}$ are given by (34).

\subsection{Results of the asymptotic expansion}

We will not try here to justify the full ansatz (11) for the eigenvector $\phi^{\varepsilon}$. Instead we will rigorously justify the result of exponential concentration postulated in (11) in the following sections with a different method, and will show that the expressions for $M, \phi^{0}, \lambda^{0}$, and $\lambda^{1}$ predicted by the asymptotic expansion are indeed correct. Let us mention that we will not seek any justifications for the higher order terms $\phi_{k}^{1}, \phi_{k l}^{2}$, and $\phi^{3}$. Indeed, it is shown in [4] in the similar case of diffusion equations that the error term $r_{\varepsilon}$ defined by

$$
\phi^{\varepsilon}(x, v)=\exp \left(-\frac{M x \cdot x}{2 \varepsilon}\right)\left(\phi^{0}\left(\frac{x}{\varepsilon}, v\right)+x_{k} \phi_{k}^{1}\left(\frac{x}{\varepsilon}, v\right)+x_{k} x_{l} \phi_{k l}^{2}\left(\frac{x}{\varepsilon}, v\right)+\varepsilon \phi^{3}\left(\frac{x}{\varepsilon}, v\right)\right)+r_{\varepsilon}
$$

is of order $\sqrt{\varepsilon}$ in any $L^{p}$ norm, and hence of the same order as the first corrector term $x_{k} \phi_{k}^{1}$.

\section{MAin RESUlts}

This section is devoted to the statement of our main result on the homogenization concentration in transport. Throughout this paper, the heterogeneous and periodic transport eigenvectors are normalized in such a way that their $L^{2}$-norm in the phase space is 1 ,

$$
\left\|\phi^{\varepsilon}\right\|_{L^{2}(\Omega \times V)}=1 \text { and }\|\psi\|_{L^{2}(Y \times V)}=1 \text {. }
$$

We also normalize $\psi^{*}$ in such a way that for all $x \in \Omega$,

$$
\int_{Y} \int_{V} \int_{V} f\left(x, y, v^{\prime}, v\right) \psi\left(x, y, v^{\prime}\right) \psi^{*}(x, y, v) \mathrm{d} y \mathrm{~d} v \mathrm{~d} v^{\prime}=1 .
$$

\subsection{The homogenized problem}

We introduce the homogenized eigenvalue problem for the transport equation (1)

$$
\left\{\begin{array}{l}
-\operatorname{div}(\bar{D} \nabla u)+(\bar{A} z \cdot z+\bar{\gamma}) u+z \cdot\left(\bar{B}^{*} \nabla u\right)=\lambda \bar{\sigma} u \\
u \in H^{1}\left(\mathbb{R}^{N}\right) \cap L_{z}^{2}\left(\mathbb{R}^{N}\right)
\end{array}\right.
$$

where $L_{z}^{2}\left(\mathbb{R}^{N}\right)=\left\{u(z) \in L^{2}\left(\mathbb{R}^{N}\right),|z| u(z) \in L^{2}\left(\mathbb{R}^{N}\right)\right\}$. This homogenized problem is a convection-diffusion problem, which is posed on the whole space $\mathbb{R}^{N}$. The homogenized coefficients are given by the following 
formulas

$$
\left\{\begin{aligned}
\bar{\sigma} & =\int_{Y} \int_{Y} \int_{V} f\left(0, y, v^{\prime}, v\right) \psi\left(0, y, v^{\prime}\right) \psi^{*}(0, y, v) \mathrm{d} y \mathrm{~d} v \mathrm{~d} v^{\prime} \\
\bar{\gamma} & =\int_{Y} \int_{V} v \cdot \nabla_{x} \psi(0, y, v) \psi^{*}(0, y, v) \mathrm{d} y \mathrm{~d} v \\
\bar{A}_{i j} & =\lambda_{i j}^{2} \int_{Y} \int_{V} \int_{V} f\left(0, y, v^{\prime}, v\right) \psi\left(0, y, v^{\prime}\right) \psi^{*}(0, y, v) \mathrm{d} y \mathrm{~d} v \mathrm{~d} v^{\prime} \\
\bar{B}_{i j} & =\int_{Y} \int_{V} v_{j} \partial_{x_{i}}\left(\psi \psi^{*}\right)(0, y, v) \mathrm{d} y \mathrm{~d} v \\
\bar{D}_{i j} & =-\int_{Y} \int_{V} v_{i} \psi^{*}(0, y, v) \chi^{j}(y, v) \mathrm{d} y \mathrm{~d} v
\end{aligned}\right.
$$

where the functions $\chi^{j}(y, v)$ are defined as the solutions of the cell problems (22), i.e.,

$$
\left\{\begin{aligned}
v \cdot \nabla_{y} \chi^{j}+\Sigma(0, y, v) \chi^{j} & =\int_{V} \sigma\left(0, y, v^{\prime}, v\right) \chi^{j}\left(y, v^{\prime}\right) \mathrm{d} v^{\prime} \\
& +\lambda^{\infty}(0) \int_{V} f\left(0, y, v^{\prime}, v\right) \chi^{j}\left(y, v^{\prime}\right) \mathrm{d} v^{\prime}-v_{j} \psi(0, y, v) \\
y \mapsto \chi^{j}(y, v) Y \text {-periodic. } &
\end{aligned}\right.
$$

According to the Fredholm alternative, since $J(0)=0$, equation (35) has a solution $\chi^{j}$, which is unique up to the addition of a multiple of $\psi(x=0)$. Because $J(0)=0$, one can easily check that adding such a multiple of $\psi$ does not change the homogenized coefficients $\bar{D}_{i j}$.

Remark 3.1. Equation (33) is well known in quantum mechanics where it is called the harmonic oscillator equation. The first eigenvector of (33) is explicitly given by (see e.g. [13])

$$
u_{1}(z)=\exp \left(-\frac{M z \cdot z}{2}\right)
$$

where, after some algebra, $M$ is the solution of the same Riccati equation (26) as in the previous section. Moreover, the corresponding first eigenvalue is

$$
\lambda^{1}=\frac{\operatorname{Tr}(M \bar{D})+\bar{\gamma}}{\bar{\sigma}},
$$

and corresponds to the first order eigenvalue corrector given by our asymptotic expansion.

Recall that Remark 2.2 states that $\bar{B}=\nabla_{x} J(0)$ where $J(x)$ is defined by (9). Therefore, if we assume that $\nabla_{x} J(0)=0$, the convection term in (33) disappear and $M$ is given by the explicit formula (30).

It is well known that the spectral problem (33) is compact in $L^{2}\left(\mathbb{R}^{N}\right)$ because of the positive quadratic potential. Remark however that (33) is usually not self-adjoint. Therefore its spectrum is made of at most a countable number of finite multiplicity eigenvalues (possibly complex-valued). We label the eigenvalues of (33) by increasing order of their real parts (with repeated multiplicity). Since (33) satisfies a maximum principle, by the Krein-Rutman theorem it admits a first eigenvalue which is real, positive, simple, and such that its eigenvector can be chosen positive in $\mathbb{R}^{N}$. In particular, this implies that the spectrum of (33) is never empty. Of course, if (33) is self-adjoint (in the case where $\bar{B}=\nabla J(0)=0$ ), then it admits a countable infinite number of real eigenvalues.

\subsection{Main result}

The main result of this paper, which justifies many of the homogenization and concentration features presented in the previous section, is as follows: 
Theorem 3.2. We assume that (H1-H4) hold. Let $\left(\lambda^{\infty}(x), \psi(x, y, v)\right)$ be the first positive eigenpair of (7). Let $\left(\lambda_{m}\right)_{1 \leq m \leq m_{\infty}}$ and $\left(\lambda_{m}^{\varepsilon}\right)_{m}$ be the eigenvalues (with repeated multiplicity and in increasing order) of the homogenized problem (33) and the original problem (1), respectively. Then, for any $m \in\left\{1, \ldots, m_{\infty}\right\}$ and for sufficiently small $\varepsilon$, there exists an $m^{\text {th }}$ eigenvalue $\lambda_{m}^{\varepsilon}$ of (1) such that

$$
\lambda_{m}^{\varepsilon}=\lambda^{\infty}(0)+\varepsilon \lambda_{m}+o(\varepsilon)
$$

and, if $\phi_{m}^{\varepsilon}$ is a corresponding normalized eigenvector of (1), then it satisfies

$$
\phi_{m}^{\varepsilon}(x, v)=\psi\left(x, \frac{x}{\varepsilon}, v\right) u_{m}^{\varepsilon}\left(\frac{x}{\sqrt{\varepsilon}}, v\right)
$$

where, up to a subsequence, $\varepsilon^{N / 4} u_{m}^{\varepsilon}(z, v)$ (properly extended to $\mathbb{R}^{N} \times V$ ) converges to $u_{m}(z)$ strongly in $L^{2}\left(\mathbb{R}^{N}\right.$ $\times V)$, and $u_{m}$ is an eigenvector associated to $\lambda_{m}$ of the homogenized convection-diffusion eigenvalue problem (33). Moreover, in the original domain we have the following convergence

$$
\lim _{\varepsilon \rightarrow 0}\left\|\phi_{m}^{\varepsilon}(x, v)-\varepsilon^{-N / 4} \psi\left(0, \frac{x}{\varepsilon}, v\right) u_{m}\left(\frac{x}{\sqrt{\varepsilon}}\right)\right\|_{L^{2}(\Omega \times V)}=0
$$

Remark 3.3. The coefficient $\varepsilon^{N / 4}$ comes from the scaling $\left\|\phi_{m}^{\varepsilon}\right\|_{L^{2}(\Omega \times V)}=1$ which implies that $\left\|\varepsilon^{N / 4} u_{m}^{\varepsilon}\right\|$ $L^{2}\left(\mathbb{R}^{N} \times V\right)$ is of order one.

The convergence of the eigenvectors is obtained up to a subsequence because of the possible multiplicity of the limit eigenvalue. Since the first eigenvalue $\lambda_{1}$ is simple, the whole sequence $\left(\lambda_{1}^{\varepsilon}, u_{1}^{\varepsilon}\right)$ converges (and not merely a subsequence).

In the sequel, we shall use the following convenient notation: for a function $g(x, y, v), Y$-periodic with respect to the fast variable $y$, we define

$$
g^{\varepsilon}(x, v)=g\left(x, \frac{x}{\varepsilon}, v\right)
$$

To prove Theorem 3.2, we first establish that the spectral problem (1) is equivalent to another problem obtained by factorization.

Proposition 3.4. Let $\psi(x, y, v)$ be the positive eigenvector of (7). Then, the linear operator

$$
\left\{\begin{aligned}
L^{2}(\Omega \times V) & \longrightarrow L^{2}(\Omega \times V) \\
\phi(x, v) & \longmapsto u(x, v)=\frac{\phi(x, v)}{\psi^{\varepsilon}(x, v)}
\end{aligned}\right.
$$

is continuous and has a continuous inverse. With this change of unknowns, the problem (1) is equivalent to the following spectral problem

$$
\left\{\begin{array}{l}
v \cdot \nabla u^{\varepsilon}+\alpha^{\varepsilon} u^{\varepsilon}+\frac{1}{\varepsilon} Q^{\varepsilon}\left(u^{\varepsilon}\right)+\frac{\lambda^{\infty}(x)-\lambda^{\infty}(0)}{\varepsilon} F^{\varepsilon}\left(u^{\varepsilon}\right)=\mu^{\varepsilon} F^{\varepsilon}\left(u^{\varepsilon}\right) \quad \text { in } \Omega \times V \\
u^{\varepsilon}=0 \quad \text { on } \Gamma_{-},
\end{array}\right.
$$


where we have defined

$$
\left\{\begin{aligned}
\mu^{\varepsilon}= & \frac{\lambda^{\varepsilon}-\lambda^{\infty}(0)}{\varepsilon} \\
Q^{\varepsilon}(u)(x, v)= & \frac{u(x, v)}{\psi^{\varepsilon}(x, v)} \int_{V} \sigma_{\infty}^{\varepsilon}\left(x, v^{\prime}, v\right) \psi^{\varepsilon}\left(x, v^{\prime}\right) \mathrm{d} v^{\prime} \\
& -\frac{1}{\psi^{\varepsilon}(x, v)} \int_{V} \sigma_{\infty}^{\varepsilon}\left(x, v^{\prime}, v\right) \psi^{\varepsilon}\left(x, v^{\prime}\right) u\left(x, v^{\prime}\right) \mathrm{d} v^{\prime} \\
F^{\varepsilon}(u)(x, v)= & \frac{1}{\psi^{\varepsilon}(x, v)} \int_{V} f^{\varepsilon}\left(x, v^{\prime}, v\right) \psi^{\varepsilon}\left(x, v^{\prime}\right) u\left(x, v^{\prime}\right) \mathrm{d} v^{\prime} \\
\alpha^{\varepsilon}(x, v)= & \frac{v \cdot\left(\nabla_{x} \psi\right)^{\varepsilon}(x, v)}{\psi^{\varepsilon}(x, v)}
\end{aligned}\right.
$$

with the notation

$$
\begin{aligned}
& \sigma_{\infty}\left(x, y, v^{\prime}, v\right)=\sigma\left(x, y, v^{\prime}, v\right)+\lambda^{\infty}(x) f\left(x, y, v^{\prime}, v\right) \\
& \sigma_{\infty}^{*}\left(x, y, v^{\prime}, v\right)=\sigma^{*}\left(x, y, v^{\prime}, v\right)+\lambda^{\infty}(x) f^{*}\left(x, y, v^{\prime}, v\right) .
\end{aligned}
$$

Proof. The result is obtained by straightforward algebra. Notice that the positivity and boundedness of $\psi$ that we stated in Proposition 1.7 are required to justify the change of unknown function (38).

We next introduce another change of variables, which will be of crucial importance to display the concentration effects,

$$
\left\{\begin{array}{l}
\Omega \longrightarrow \Omega^{\varepsilon}=\varepsilon^{-1 / 2} \Omega \\
x \longmapsto z=\frac{x}{\sqrt{\varepsilon}}
\end{array}\right.
$$

For each function $g(x, y, v), Y$-periodic with respect to the fast variable $y$, we introduce the notation

$$
\tilde{g}^{\varepsilon}(z, v)=g\left(\sqrt{\varepsilon} z, \frac{z}{\sqrt{\varepsilon}}, v\right) \text {, with } z=\frac{x}{\sqrt{\varepsilon}} \in \Omega^{\varepsilon} .
$$

We similarly define the operators $\tilde{Q}^{\varepsilon}$ and $\tilde{F}^{\varepsilon}$ from $Q^{\varepsilon}$ and $F^{\varepsilon}$. For instance, with this notation we have

$$
\nabla\left(\psi^{\varepsilon}\right)(x, v)=\left(\nabla_{x} \psi+\frac{1}{\varepsilon} \nabla_{y} \psi\right)\left(x, \frac{x}{\varepsilon}, v\right)=\left(\left(\nabla_{x} \psi\right)^{\varepsilon}+\frac{1}{\varepsilon}\left(\nabla_{y} \psi\right)^{\varepsilon}\right)(x, v),
$$

and

$$
\nabla\left(\tilde{\psi}^{\varepsilon}\right)(z, v)=\left(\sqrt{\varepsilon} \nabla_{x} \psi+\frac{1}{\sqrt{\varepsilon}} \nabla_{y} \psi\right)\left(\sqrt{\varepsilon} z, \frac{z}{\sqrt{\varepsilon}}, v\right)=\left(\sqrt { \varepsilon } \left(\widetilde{\left.\nabla_{x} \psi\right)^{\varepsilon}}+\frac{1}{\sqrt{\varepsilon}}\left(\widetilde{\left.\nabla_{y} \psi\right)^{\varepsilon}}\right)(z, v) .\right.\right.
$$

Accordingly we obtain the following result:

Proposition 3.5. With the change of variables (42), the spectral equation (39) becomes

$$
\left\{\begin{array}{l}
\frac{1}{\sqrt{\varepsilon}} v \cdot \nabla \tilde{u}^{\varepsilon}+\tilde{\alpha}^{\varepsilon} \tilde{u}^{\varepsilon}+\frac{1}{\varepsilon} \tilde{Q}^{\varepsilon}\left(\tilde{u}^{\varepsilon}\right)+\frac{\lambda^{\infty}(\sqrt{\varepsilon} z)-\lambda^{\infty}(0)}{\varepsilon} \tilde{F}^{\varepsilon}\left(\tilde{u}^{\varepsilon}\right)=\mu^{\varepsilon} \tilde{F}^{\varepsilon}\left(\tilde{u}^{\varepsilon}\right) \text { in } \Omega^{\varepsilon} \times V, \\
\tilde{u}^{\varepsilon}=0 \quad \text { on } \Gamma_{-}^{\varepsilon} .
\end{array}\right.
$$

The spectral equation (43) is recast as

$$
S^{\varepsilon} \tilde{u}^{\varepsilon}=\frac{1}{\mu^{\varepsilon}+\eta} \tilde{u}^{\varepsilon}
$$


Here, the compact (see $[2,6])$ operator $S^{\varepsilon}$ is defined by

$$
S^{\varepsilon}:\left\{\begin{aligned}
L^{2}\left(\Omega^{\varepsilon} \times V\right) & \longrightarrow L^{2}\left(\Omega^{\varepsilon} \times V\right) \\
\tilde{q}(z, v) & \longmapsto \tilde{u}^{\varepsilon}(z, v)
\end{aligned}\right.
$$

where $\tilde{u}^{\varepsilon}$ denotes from now on the solution of the following source problem associated to (39)

$$
\left\{\begin{array}{l}
\frac{1}{\sqrt{\varepsilon}} v \cdot \nabla \tilde{u}^{\varepsilon}+\tilde{\alpha}^{\varepsilon} \tilde{u}^{\varepsilon}+\frac{1}{\varepsilon} \tilde{Q}^{\varepsilon}\left(\tilde{u}^{\varepsilon}\right)+\left(\frac{\lambda^{\infty}(\sqrt{\varepsilon} z)-\lambda^{\infty}(0)}{\varepsilon}+\eta\right) \tilde{F}^{\varepsilon}\left(\tilde{u}^{\varepsilon}\right)=\tilde{F}^{\varepsilon}(\tilde{q}) \quad \text { in } \Omega^{\varepsilon} \times V \\
\tilde{u}^{\varepsilon}=0 \text { on } \Gamma_{-}^{\varepsilon} .
\end{array}\right.
$$

Notice the presence of a positive coefficient $\eta>0$ in equations (44) and (46). This coefficient will be useful in our energy estimates and is harmless because it simply shifts the eigenvalues of (43) to the right. The sequence $\tilde{u}^{\varepsilon}$ is defined on domains $\Omega^{\varepsilon} \times V$ that depend on $\varepsilon$. To establish a convergence proof, we need to extend $\tilde{u}^{\varepsilon}$ to $\mathbb{R}^{N} \times V$ as follows. We assume that $\tilde{u}^{\varepsilon}$ solves

$$
v \cdot \nabla \tilde{u}^{\varepsilon}+\mathrm{e}^{\frac{1}{\varepsilon}} \tilde{u}^{\varepsilon}=0 \quad \text { on } \quad\left(\mathbb{R}^{N} \backslash \Omega^{\varepsilon}\right) \times V
$$

and impose the continuity of $\tilde{u}^{\varepsilon}(x, v)$ across the interface $\partial \Omega^{\varepsilon} \times V$. We also assume that no particles arrive from infinity, i.e., $\tilde{u}^{\varepsilon}(x, v) \rightarrow 0$ as $|x| \rightarrow \infty$.

Theorem 3.6. Under the hypotheses of Theorem 3.2, the sequence $\tilde{u}^{\varepsilon}(z, v)$ of solutions of (46) converges strongly in $L^{2}\left(\mathbb{R}^{N} \times V\right)$ to $u(z)$, the solution of the following homogenized problem

$$
\left\{\begin{array}{l}
-\operatorname{div}(\bar{D} \nabla u)+(\bar{A} z . z+\eta \bar{\sigma}+\bar{\gamma}) u+z \cdot\left(\bar{B}^{*} \nabla u\right)=\overline{F(\tilde{q})} \\
u \in H^{1}\left(\mathbb{R}^{N}\right) \cap L_{z}^{2}\left(\mathbb{R}^{N}\right)
\end{array}\right.
$$

where $\bar{D}, \bar{A}, \bar{B}, \bar{\sigma}$, and $\bar{\gamma}$ are given in (34) and

$$
\overline{F(\tilde{q})}=\int_{Y} \int_{V} \int_{V} f\left(0, y, v^{\prime}, v\right) \psi\left(0, y, v^{\prime}\right) q\left(z, v^{\prime}\right) \psi^{*}(0, y, v) \mathrm{d} y \mathrm{~d} v \mathrm{~d} v^{\prime} .
$$

This theorem will be proved in Sections 4 and 5 .

\subsection{Proof of Theorem 3.2}

We are now in a position to prove our main result. Let us define the homogenized operator $S$ by

$$
S:\left\{\begin{aligned}
L^{2}\left(\mathbb{R}^{N} \times V\right) & \longrightarrow L^{2}\left(\mathbb{R}^{N} \times V\right) \\
\tilde{q}(z, v) & \longmapsto u(z)
\end{aligned}\right.
$$

where $u$ is the solution of the homogenized equation (48). Then $S$ is a compact operator because $H^{1}\left(\mathbb{R}^{N}\right)$ $\cap L_{z}^{2}\left(\mathbb{R}^{N}\right)$ is compactly embedded in $L^{2}\left(\mathbb{R}^{N}\right)$. We deduce from Theorem 3.6 that $S^{\varepsilon}$ converges to $S$ pointwise in $L^{2}\left(\mathbb{R}^{N} \times V\right)$, in the sense that for all $q \in L^{2}\left(\mathbb{R}^{N} \times V\right)$, then $S^{\varepsilon}(q) \rightarrow S(q)$ in $L^{2}\left(\mathbb{R}^{N} \times V\right)$ strongly.

Furthermore, as a consequence of Corollary $4.3, S^{\varepsilon}$ converges compactly to $S$, in the sense that, for every bounded sequence $\tilde{q}^{\varepsilon}$ in $L^{2}\left(\mathbb{R}^{N} \times V\right), S^{\varepsilon}\left(\tilde{q}^{\varepsilon}\right)$ is relatively compact in $L^{2}\left(\mathbb{R}^{N} \times V\right)$. The following classical result in operator theory, recalled here for completeness (see $[5,10]$ ), allows us to conclude that the spectrum of $S^{\varepsilon}$ converges to that of $S$. Eventually, estimate (37) is due to the special form of the eigenfunctions of the homogenized problem (33), which are exponentially decaying away from the concentration point 0 , thus allowing to replace $\psi(x, x / \varepsilon, v)$ by $\psi(0, x / \varepsilon, v)$ in the factorization $(36)$. 
Theorem 3.7. Let $X$ be a Banach space, and $\left(T_{n}\right)_{n \in \mathbb{N}}$ a sequence of bounded operators in $\mathcal{L}(X)$ converging compactly to $T$. Let $\sigma(T)$ and $\sigma\left(T_{n}\right)$ be the spectra of $T$ and $T_{n}$ respectively. Let $\lambda$ be an isolated eigenvalue of $T$ of finite (algebraic) multiplicity $m$ and let $\Gamma$ be a closed Jordan curve in the complex plane enclosing $\lambda$ and leaving outside the rest of the spectrum of $T$. Then, for sufficiently large values of $n$, $\Gamma$ encloses exactly $m$ eigenvalues of $T_{n}$ (with repeated algebraic multiplicity).

Moreover, if $\lambda_{n}$ is a sequence of eigenvalues of $T_{n}$ converging to $\lambda$, and $u_{n}$ is a sequence of normalized associated eigenvectors, then, up to a subsequence, $u_{n}$ converges to a limit $u$ in $X$ which is an eigenvector of $T$ associated with $\lambda$.

\section{A PRIORI ESTIMATES}

The first step in the proof of Theorem 3.6 is to derive a priori energy estimates for the source problem (46). These estimates are as follows:

Lemma 4.1. Let $\tilde{u}^{\varepsilon}$ be the unique solution of (46). Then there exists a positive constant $C$ independent of $\varepsilon$ and $\tilde{q}$, such that

$$
\begin{gathered}
\left\|\tilde{u}^{\varepsilon}\right\|_{L^{2}\left(\Omega^{\varepsilon} \times V\right)}+\left\|v \cdot \nabla \tilde{u}^{\varepsilon}\right\|_{L^{2}\left(\Omega^{\varepsilon} \times V\right)}+\left\||z| \int_{V} \tilde{u}^{\varepsilon}\right\|_{L^{2}\left(\Omega^{\varepsilon} \times V\right)} \\
+\frac{1}{\sqrt{\varepsilon}}\left\|\tilde{u}^{\varepsilon}-\int_{V} \tilde{u}^{\varepsilon}\right\|_{L^{2}\left(\Omega^{\varepsilon} \times V\right)}+\frac{1}{\varepsilon^{\frac{1}{4}}}\left\|\tilde{u}^{\varepsilon}\right\|_{L^{2}\left(\Gamma_{+}^{\varepsilon},|v \cdot n|\right)} \leq C\|\tilde{q}\|_{L^{2}\left(\Omega^{\varepsilon} \times V\right)}
\end{gathered}
$$

where $L^{2}\left(\Gamma_{+}^{\varepsilon},|v \cdot n|\right)$ is the trace space of functions u satisfying $\int_{\Gamma_{+}^{\varepsilon}}(v \cdot n)|u|^{2} \mathrm{~d} \Gamma<\infty$ with $\Gamma_{+}^{\varepsilon}=\{(x, v) \in$ $\left.\partial \Omega^{\varepsilon} \times V \mid v \cdot n(x)>0\right\}$ and $\mathrm{d} \Gamma=\mathrm{d} v \mathrm{~d} \sigma$ (d $\sigma$ being the surface measure on $\left.\partial \Omega^{\varepsilon}\right)$.

Proof. We multiply equation (46) by $\tilde{u}^{\varepsilon} \tilde{\psi}^{\varepsilon} \tilde{\psi}^{* \varepsilon}$, taking into account the notation (40), and integrate over $\Omega^{\varepsilon} \times V$ to obtain

$$
\begin{aligned}
& \int_{\Omega^{\varepsilon}} \int_{V} \frac{1}{\sqrt{\varepsilon}} v \cdot \nabla \tilde{u}^{\varepsilon} \tilde{u}^{\varepsilon} \tilde{\psi}^{\varepsilon} \tilde{\psi}^{* \varepsilon}+v \cdot\left(\widetilde{\nabla_{x} \psi}\right)^{\varepsilon}\left(\tilde{u}^{\varepsilon}\right)^{2} \tilde{\psi}^{* \varepsilon}+\frac{1}{\varepsilon} \tilde{Q}^{\varepsilon}\left(\tilde{u}^{\varepsilon}\right) \tilde{u}^{\varepsilon} \tilde{\psi}^{\varepsilon} \tilde{\psi}^{* \varepsilon} \\
& +\left(\frac{\lambda_{\infty}(\sqrt{\varepsilon} z)-\lambda_{\infty}(0)}{\varepsilon}+\eta\right) \tilde{F}^{\varepsilon}\left(\tilde{u}^{\varepsilon}\right) \tilde{u}^{\varepsilon} \tilde{\psi}^{\varepsilon} \tilde{\psi}^{* \varepsilon} \mathrm{d} z \mathrm{~d} v=\int_{\Omega^{\varepsilon}} \int_{V} \tilde{F}^{\varepsilon}(\tilde{q}) \tilde{u}^{\varepsilon} \tilde{\psi}^{\varepsilon} \tilde{\psi}^{* \varepsilon} \mathrm{d} z \mathrm{~d} v
\end{aligned}
$$

Let $I_{1}=\frac{1}{\sqrt{\varepsilon}} \int_{\Omega^{\varepsilon}} \int_{V} v \cdot \nabla \tilde{u}^{\varepsilon} \tilde{u}^{\varepsilon} \tilde{\psi}^{\varepsilon} \tilde{\psi}^{* \varepsilon} \mathrm{d} z \mathrm{~d} v$. Then we have

$$
\begin{aligned}
I_{1}= & \frac{1}{\sqrt{\varepsilon}} \int_{\Gamma_{+}^{\varepsilon}}(v \cdot n)\left(\tilde{u}^{\varepsilon}\right)^{2} \tilde{\psi}^{\varepsilon} \tilde{\psi}^{* \varepsilon} \mathrm{d} z \mathrm{~d} v-\frac{1}{\sqrt{\varepsilon}} \int_{\Omega^{\varepsilon}} \int_{V} v \cdot \nabla\left(\tilde{u}^{\varepsilon} \tilde{\psi}^{\varepsilon} \tilde{\psi}^{* \varepsilon}\right) \tilde{u}^{\varepsilon} \mathrm{d} z \mathrm{~d} v \\
= & \frac{1}{\sqrt{\varepsilon}} \int_{\Gamma_{+}^{\varepsilon}}(v \cdot n)\left(\tilde{u}^{\varepsilon}\right)^{2} \tilde{\psi}^{\varepsilon} \tilde{\psi}^{* \varepsilon} \mathrm{d} z \mathrm{~d} v-\frac{1}{\sqrt{\varepsilon}} \int_{\Omega^{\varepsilon}} \int_{V} v \cdot \nabla \tilde{u}^{\varepsilon} \tilde{u}^{\varepsilon} \tilde{\psi}^{\varepsilon} \tilde{\psi}^{* \varepsilon} \mathrm{d} z \mathrm{~d} v \\
& -\frac{1}{\sqrt{\varepsilon}} \int_{\Omega^{\varepsilon}} \int_{V} v \cdot \nabla\left(\tilde{\psi}^{\varepsilon} \tilde{\psi}^{* \varepsilon}\right)\left(\tilde{u}^{\varepsilon}\right)^{2} \mathrm{~d} z \mathrm{~d} v \\
= & \frac{1}{2 \sqrt{\varepsilon}} \int_{\Gamma_{+}^{\varepsilon}}(v \cdot n)\left(\tilde{u}^{\varepsilon}\right)^{2} \tilde{\psi}^{\varepsilon} \tilde{\psi}^{* \varepsilon} \mathrm{d} z \mathrm{~d} v-\frac{1}{2 \sqrt{\varepsilon}} \int_{\Omega^{\varepsilon}} \int_{V} v \cdot \nabla\left(\tilde{\psi}^{\varepsilon} \tilde{\psi}^{* \varepsilon}\right)\left(\tilde{u}^{\varepsilon}\right)^{2} \mathrm{~d} z \mathrm{~d} v \\
= & \frac{1}{2 \sqrt{\varepsilon}} \int_{\Gamma_{+}^{\varepsilon}}(v \cdot n)\left(\tilde{u}^{\varepsilon}\right)^{2} \tilde{\psi}^{\varepsilon} \tilde{\psi}^{* \varepsilon} \mathrm{d} z \mathrm{~d} v-\frac{1}{2} \int_{\Omega^{\varepsilon}} \int_{V} v \cdot\left(\widetilde{\nabla_{x} \psi \psi^{*}}\right)^{\varepsilon}\left(\tilde{u}^{\varepsilon}\right)^{2} \mathrm{~d} z \mathrm{~d} v \\
& -\frac{1}{2 \varepsilon} \int_{\Omega^{\varepsilon}} \int_{V} v \cdot\left(\widetilde{\nabla_{y} \psi \psi^{*}}\right)^{\varepsilon}\left(\tilde{u}^{\varepsilon}\right)^{2} \mathrm{~d} z \mathrm{~d} v .
\end{aligned}
$$


Upon multiplying the infinite medium equation (7) by $\psi^{*}$ and subtracting the adjoint equation (8) multiplied by $\psi$, we get

$$
v \cdot \nabla_{y}\left(\psi \psi^{*}\right)=\psi^{*} \int_{V} \sigma_{\infty} \psi \mathrm{d} v^{\prime}-\psi \int_{V} \sigma_{\infty}^{*} \psi^{*} \mathrm{~d} v^{\prime}
$$

where $\sigma_{\infty}$ and $\sigma_{\infty}^{*}$ are defined in (41). Thus, it yields the following expression for $I_{1}$

$$
\begin{aligned}
I_{1}= & \frac{1}{2 \sqrt{\varepsilon}} \int_{\Gamma_{+}^{\varepsilon}}(v \cdot n)\left(\tilde{u}^{\varepsilon}\right)^{2} \tilde{\psi}^{\varepsilon} \tilde{\psi}^{* \varepsilon} \mathrm{d} z \mathrm{~d} v-\frac{1}{2} \int_{\Omega^{\varepsilon}} \int_{V} v \cdot \widetilde{\nabla_{x} \psi \psi^{*}}\left(\tilde{u}^{\varepsilon}\right)^{2} \mathrm{~d} z \mathrm{~d} v \\
& -\frac{1}{2 \varepsilon} \int_{\Omega^{\varepsilon}} \int_{V}\left(\tilde{u}^{\varepsilon}\right)^{2}\left(\tilde{\psi}^{* \varepsilon} \int_{V} \tilde{\sigma}_{\infty}^{\varepsilon} \tilde{\psi}^{\varepsilon} \mathrm{d} v^{\prime}-\tilde{\psi}^{\varepsilon} \int_{V} \tilde{\sigma}_{\infty}^{* \varepsilon} \tilde{\psi}^{* \varepsilon} d v^{\prime}\right) \mathrm{d} z \mathrm{~d} v .
\end{aligned}
$$

Let $I_{2}=\frac{1}{\varepsilon} \int_{\Omega^{\varepsilon}} \int_{V} \tilde{Q}^{\varepsilon}\left(\tilde{u}^{\varepsilon}\right) \tilde{u}^{\varepsilon} \tilde{\psi}^{\varepsilon} \tilde{\psi}^{* \varepsilon} \mathrm{d} z \mathrm{~d} v$. Then,

$$
I_{2}=\frac{1}{\varepsilon} \int_{\Omega^{\varepsilon}} \int_{V}\left(\left(\tilde{u}^{\varepsilon}\right)^{2} \tilde{\psi}^{* \varepsilon} \int_{V} \tilde{\sigma}_{\infty}^{\varepsilon} \tilde{\psi}^{\varepsilon} \mathrm{d} v^{\prime}-\tilde{u}^{\varepsilon} \tilde{\psi}^{* \varepsilon} \int_{V} \tilde{\sigma}_{\infty}^{\varepsilon} \tilde{\psi}^{\varepsilon} \tilde{u}^{\varepsilon} \mathrm{d} v^{\prime}\right) \mathrm{d} z \mathrm{~d} v
$$

We deduce that

$$
\begin{aligned}
I_{1}+I_{2}= & \frac{1}{2 \sqrt{\varepsilon}} \int_{\Gamma^{\varepsilon}}(v \cdot n)\left(\tilde{u}^{\varepsilon}\right)^{2} \tilde{\psi}^{\varepsilon} \tilde{\psi}^{* \varepsilon} \mathrm{d} z \mathrm{~d} v-\frac{1}{2} \int_{\Omega^{\varepsilon}} \int_{V} v \cdot \widetilde{\nabla_{x} \psi \psi^{*}}\left(\tilde{u}^{\varepsilon}\right)^{2} \mathrm{~d} z \mathrm{~d} v \\
& +\frac{1}{2 \varepsilon} \int_{\Omega^{\varepsilon}} \int_{V}\left(\left(\tilde{u}^{\varepsilon}\right)^{2} \tilde{\psi}^{* \varepsilon} \int_{V} \tilde{\sigma}_{\infty}^{\varepsilon} \tilde{\psi}^{\varepsilon} \mathrm{d} v^{\prime}\right. \\
& \left.-2 \tilde{u}^{\varepsilon} \tilde{\psi}^{* \varepsilon} \int_{V} \tilde{\sigma}_{\infty}^{\varepsilon} \tilde{\psi}^{\varepsilon} \tilde{u}^{\varepsilon} \mathrm{d} v^{\prime}+\left(\tilde{u}^{\varepsilon}\right)^{2} \tilde{\psi}^{\varepsilon} \int_{V} \tilde{\sigma}_{\infty}^{* \varepsilon} \tilde{\psi}^{* \varepsilon} \mathrm{d} v^{\prime}\right) \mathrm{d} z \mathrm{~d} v
\end{aligned}
$$

and the third term in $I_{1}+I_{2}$ is equal to

$$
\begin{aligned}
& \frac{1}{2 \varepsilon} \int_{\Omega^{\varepsilon}} \int_{V} \int_{V} \tilde{u}^{\varepsilon}(z, v)^{2} \tilde{\psi}^{* \varepsilon}(z, v) \tilde{\sigma}_{\infty}^{\varepsilon}\left(z, v^{\prime}, v\right) \tilde{\psi}^{\varepsilon}\left(z, v^{\prime}\right)-2 \tilde{u}^{\varepsilon}(z, v) \tilde{\psi}^{* \varepsilon}(z, v) \tilde{\sigma}_{\infty}^{\varepsilon}\left(z, v^{\prime}, v\right) \tilde{\psi}^{\varepsilon}\left(z, v^{\prime}\right) \tilde{u}^{\varepsilon}\left(z, v^{\prime}\right) \\
& +\tilde{u}^{\varepsilon}\left(z, v^{\prime}\right)^{2} \tilde{\psi}^{\varepsilon}\left(z, v^{\prime}\right) \tilde{\sigma}_{\infty}^{* \varepsilon}\left(z, v, v^{\prime}\right) \tilde{\psi}^{* \varepsilon}(z, v) \mathrm{d} z \mathrm{~d} v \mathrm{~d} v^{\prime} \\
= & \frac{1}{2 \varepsilon} \int_{\Omega^{\varepsilon}} \int_{V} \int_{V} \tilde{\psi}^{* \varepsilon}(z, v) \tilde{\psi}^{\varepsilon}\left(z, v^{\prime}\right) \tilde{\sigma}_{\infty}^{\varepsilon}\left(z, v^{\prime}, v\right)\left|\tilde{u}^{\varepsilon}(z, v)-\tilde{u}^{\varepsilon}\left(z, v^{\prime}\right)\right|^{2} \mathrm{~d} z \mathrm{~d} v \mathrm{~d} v^{\prime} .
\end{aligned}
$$

At last, we find

$$
\begin{aligned}
& I_{1}+I_{2}=\frac{1}{2 \sqrt{\varepsilon}} \int_{\Gamma_{+}^{\varepsilon}}(v \cdot n)\left(\tilde{u}^{\varepsilon}\right)^{2} \tilde{\psi}^{\varepsilon} \tilde{\psi}^{* \varepsilon} \mathrm{d} z \mathrm{~d} v-\frac{1}{2} \int_{\Omega^{\varepsilon}} \int_{V} v \cdot{\widetilde{\nabla_{x} \psi \psi^{*}}}^{\varepsilon}\left(\tilde{u}^{\varepsilon}\right)^{2} \mathrm{~d} z \mathrm{~d} v \\
& +\frac{1}{2 \varepsilon} \int_{\Omega^{\varepsilon}}^{+} \int_{V} \int_{V} \tilde{\psi}^{* \varepsilon}(z, v) \tilde{\psi}^{\varepsilon}\left(z, v^{\prime}\right) \tilde{\sigma}_{\infty}^{\varepsilon}\left(z, v^{\prime}, v\right)\left|\tilde{u}^{\varepsilon}(z, v)-\tilde{u}^{\varepsilon}\left(z, v^{\prime}\right)\right|^{2} \mathrm{~d} z \mathrm{~d} v \mathrm{~d} v^{\prime},
\end{aligned}
$$

and it is straightforward to check that

$$
I_{1}+I_{2} \geq-C \int_{\Omega^{\varepsilon}} \int_{V}\left(\tilde{u}^{\varepsilon}\right)^{2} \mathrm{~d} z \mathrm{~d} v+\frac{C}{2 \varepsilon} \int_{\Omega^{\varepsilon}} \int_{V}\left|\tilde{u}^{\varepsilon}-\int_{V} \tilde{u}^{\varepsilon}\right|^{2} \mathrm{~d} z \mathrm{~d} v+\frac{C}{\sqrt{\varepsilon}} \int_{\Gamma_{\varepsilon}^{+}}(v \cdot n)\left(\tilde{u}^{\varepsilon}\right)^{2} .
$$

Let $I_{3}=\int_{\Omega^{\varepsilon}} \int_{V} v \cdot{\widetilde{\nabla_{x}}}^{\varepsilon}\left(\tilde{u}^{\varepsilon}\right)^{2} \tilde{\psi}^{* \varepsilon}+\eta \tilde{F}^{\varepsilon}\left(\tilde{u}^{\varepsilon}\right) \tilde{u}^{\varepsilon} \tilde{\psi}^{\varepsilon} \tilde{\psi}^{* \varepsilon} \mathrm{d} z \mathrm{~d} v$. Adding and subtracting the contribution $\eta \int_{\Omega^{\varepsilon}} \int_{V}\left(\tilde{u}^{\varepsilon}\right)^{2}$ $\tilde{\psi}^{* \varepsilon} \int_{V} \tilde{f}^{\varepsilon} \tilde{\psi}^{\varepsilon}$ yields

$$
I_{3}=\int_{\Omega^{\varepsilon}} \int_{V}\left(v \cdot{\widetilde{\nabla_{x}}}^{\varepsilon}+\eta \int_{V} \tilde{f}^{\varepsilon} \tilde{\psi}^{\varepsilon} \mathrm{d} v^{\prime}\right) \tilde{\psi}^{* \varepsilon}\left(\tilde{u}^{\varepsilon}\right)^{2}+\eta \tilde{u}^{\varepsilon} \tilde{\psi}^{* \varepsilon}\left(\int_{V} \tilde{f}^{\varepsilon} \tilde{\psi}^{\varepsilon} \tilde{u}^{\varepsilon} \mathrm{d} v^{\prime}-\tilde{u}^{\varepsilon} \int_{V} \tilde{f}^{\varepsilon} \tilde{\psi}^{\varepsilon} \mathrm{d} v^{\prime}\right) \mathrm{d} z \mathrm{~d} v
$$


Since $\tilde{f}^{\varepsilon}, \tilde{\psi}^{\varepsilon}$ and $\tilde{\psi}^{* \varepsilon}$ are bounded from below by positive constants, choosing a sufficiently large value of $\eta$ (which is independent of $\varepsilon$ and $q$ ), we can estimate the first term in $I_{3}$ from below by

$$
C(\eta) \int_{\Omega^{\varepsilon}} \int_{V}\left(\tilde{u}^{\varepsilon}\right)^{2} \mathrm{~d} z \mathrm{~d} v .
$$

The second term in $I_{3}$ is given by

$\eta \int_{\Omega^{\varepsilon}} \int_{V} \tilde{u}^{\varepsilon} \tilde{\psi}^{\varepsilon} \tilde{\psi}^{* \varepsilon}\left(\tilde{F}^{\varepsilon}\left(\tilde{u}^{\varepsilon}\right)-\tilde{u}^{\varepsilon} \tilde{F}^{\varepsilon}(1)\right) \mathrm{d} z \mathrm{~d} v=\eta \int_{\Omega^{\varepsilon}} \int_{V} \tilde{u}^{\varepsilon} \tilde{\psi}^{\varepsilon} \tilde{\psi}^{* \varepsilon}\left(\tilde{F}^{\varepsilon}\left(\tilde{u}^{\varepsilon}-\int_{V} \tilde{u}^{\varepsilon}\right)-\tilde{F}^{\varepsilon}(1)\left(\tilde{u}^{\varepsilon}-\int_{V} \tilde{u}^{\varepsilon}\right)\right) \mathrm{d} z \mathrm{~d} v$.

Its sign is not known a priori, but this term is bounded in absolute value by

$$
C\left\|\tilde{u}^{\varepsilon}\right\|_{L^{2}\left(\Omega^{\varepsilon} \times V\right)}\left\|\tilde{u}^{\varepsilon}-\int_{V} \tilde{u}^{\varepsilon}\right\|_{L^{2}\left(\Omega^{\varepsilon} \times V\right)} .
$$

Let us define $I_{4}=\int_{\Omega^{\varepsilon}} \int_{V} \varepsilon^{-1}\left(\lambda^{\infty}(\sqrt{\varepsilon} z)-\lambda^{\infty}(0)\right) \tilde{F}^{\varepsilon}\left(\tilde{u}^{\varepsilon}\right) \tilde{u}^{\varepsilon} \tilde{\psi}^{\varepsilon} \tilde{\psi}^{* \varepsilon} \mathrm{d} z \mathrm{~d} v$. According to the hypotheses on the function $x \mapsto \lambda^{\infty}(x)$, it is clear that $\lambda^{\infty}(x)-\lambda^{\infty}(0)$ is bounded from below on $\Omega$ by a quadratic positive definite form, i.e.,

$$
\exists C>0, \forall x \in \Omega, \lambda^{\infty}(x)-\lambda^{\infty}(0) \geq C x \cdot x .
$$

Since $\tilde{f}^{\varepsilon}, \tilde{\psi}^{\varepsilon}$ and $\tilde{\psi}^{* \varepsilon}$ are also bounded from below by positive constants, and $V$ is bounded according to (H1), we deduce that

$$
I_{4} \geq C \int_{\Omega^{\varepsilon}} \int_{V}|z|^{2} \tilde{u}^{\varepsilon} \tilde{\psi}^{* \varepsilon}\left(\int_{V} \tilde{u}^{\varepsilon} \tilde{f}^{\varepsilon} \tilde{\psi}^{\varepsilon} \mathrm{d} v^{\prime}\right) \mathrm{d} z \mathrm{~d} v \geq C \int_{\Omega^{\varepsilon}}\left(|z| \int_{V} \tilde{u}^{\varepsilon} \mathrm{d} v^{\prime}\right)^{2} \mathrm{~d} z
$$

Finally, the right-hand side in (51) is equal to $\int_{\Omega^{\varepsilon}} \int_{V} \tilde{u}^{\varepsilon} \tilde{\psi}^{* \varepsilon}\left(\int_{V} \tilde{f}^{\varepsilon} \tilde{\psi}^{\varepsilon} \tilde{q}\right) \mathrm{d} z \mathrm{~d} v \mathrm{~d} v^{\prime}$, hence is bounded by

$$
C\left\|\tilde{u}^{\varepsilon}\right\|_{L^{2}\left(\Omega^{\varepsilon} \times V\right)}\|\tilde{q}\|_{L^{2}\left(\Omega^{\varepsilon} \times V\right)} .
$$

Summing up the estimates in $(53,55-57)$, and (58), we deduce that

$$
\begin{aligned}
& \quad C\left\|\tilde{u}^{\varepsilon}\right\|_{L^{2}\left(\Omega^{\varepsilon} \times V\right)}^{2}+\frac{C}{\varepsilon}\left\|\tilde{u}^{\varepsilon}-\int_{V} \tilde{u}^{\varepsilon}\right\|_{L^{2}\left(\Omega^{\varepsilon} \times V\right)}^{2}+\frac{C}{\sqrt{\varepsilon}}\left\|\tilde{u}^{\varepsilon}\right\|_{L^{2}\left(\Gamma_{\varepsilon}^{+},|v \cdot n|\right)}^{2}+C\left\||z| \int_{V} \tilde{u}^{\varepsilon}\right\|_{L^{2}\left(\Omega^{\varepsilon} \times V\right)}^{2} \\
& -C\left\|\tilde{u}^{\varepsilon}\right\|_{L^{2}\left(\Omega^{\varepsilon} \times V\right)}\left\|\tilde{u}^{\varepsilon}-\int_{V} \tilde{u}^{\varepsilon}\right\|_{L^{2}\left(\Omega^{\varepsilon} \times V\right)}+C\left\|\tilde{u}^{\varepsilon}\right\|_{L^{2}\left(\Omega^{\varepsilon} \times V\right)}^{2}\|\tilde{q}\|_{L^{2}\left(\Omega^{\varepsilon} \times V\right)} . \\
& \leq C
\end{aligned}
$$

Consequently, we have

$$
\frac{C}{\varepsilon}\left(\left\|\tilde{u}^{\varepsilon}-\int_{V} \tilde{u}^{\varepsilon}\right\|-\varepsilon\left\|\tilde{u}^{\varepsilon}\right\|\right)^{2}+(C-C \varepsilon)\left\|\tilde{u}^{\varepsilon}\right\|^{2}+C\left\||z| \int_{V} \tilde{u}^{\varepsilon}\right\|^{2} \leq C\left\|\tilde{u}^{\varepsilon}\right\|\|\tilde{q}\| .
$$

This implies first that $\left\|\tilde{u}^{\varepsilon}\right\|_{L^{2}\left(\Omega^{\varepsilon} \times V\right)} \leq C\|\tilde{q}\|_{L^{2}\left(\Omega^{\varepsilon} \times V\right)}$, and then,

$$
\left\||z| \int_{V} \tilde{u}^{\varepsilon}\right\|_{L^{2}\left(\Omega^{\varepsilon} \times V\right)} \leq C\|\tilde{q}\|_{L^{2}\left(\Omega^{\varepsilon} \times V\right)} .
$$


Next we observe that

$$
C\left(\left\|\tilde{u}^{\varepsilon}\right\|-\left\|\tilde{u}^{\varepsilon}-\int_{V} \tilde{u}^{\varepsilon}\right\|\right)^{2}+\left(\frac{C}{\varepsilon}-C\right)\left\|\tilde{u}^{\varepsilon}-\int_{V} \tilde{u}^{\varepsilon}\right\|^{2} \leq C\left\|\tilde{u}^{\varepsilon}\right\|\|\tilde{q}\|,
$$

which gives us

$$
\frac{1}{\sqrt{\varepsilon}}\left\|\tilde{u}^{\varepsilon}-\int_{V} \tilde{u}^{\varepsilon}\right\|_{L^{2}\left(\Omega^{\varepsilon} \times V\right)} \leq C\|\tilde{q}\|_{L^{2}\left(\Omega^{\varepsilon} \times V\right)} .
$$

Finally, the bound for $\left\|v \cdot \nabla \tilde{u}^{\varepsilon}\right\|_{L^{2}\left(\Omega^{\varepsilon} \times V\right)}$ is deduced from equation (46) since

$$
v \cdot \nabla \tilde{u}^{\varepsilon}=-\sqrt{\varepsilon} \frac{v \cdot{\widetilde{\nabla_{x} \psi}}^{\varepsilon}}{\tilde{\psi}^{\varepsilon}} \tilde{u}^{\varepsilon}-\frac{1}{\sqrt{\varepsilon}} \tilde{Q}^{\varepsilon}\left(\tilde{u}^{\varepsilon}\right)-\left(\frac{\lambda^{\infty}(\sqrt{\varepsilon} z)-\lambda^{\infty}(0)}{\sqrt{\varepsilon}}+\sqrt{\varepsilon} \eta\right) \tilde{F}^{\varepsilon}\left(\tilde{u}^{\varepsilon}\right)+\sqrt{\varepsilon} \tilde{F}^{\varepsilon}(\tilde{q}) .
$$

The first and fourth term on the right-hand side are easily bounded by $\sqrt{\varepsilon} C\left\|\tilde{u}^{\varepsilon}\right\|$ and $\sqrt{\varepsilon} C\|\tilde{q}\|$ and hence by $\sqrt{\varepsilon} C$. Since $\tilde{Q}^{\varepsilon}\left(\tilde{u}^{\varepsilon}\right)=\tilde{Q}^{\varepsilon}\left(\tilde{u}^{\varepsilon}-\int_{V} \tilde{u}^{\varepsilon}\right)$, the second term is bounded by $\frac{1}{\sqrt{\varepsilon}} C\left\|\tilde{u}^{\varepsilon}-\int_{V} \tilde{u}^{\varepsilon}\right\|$ and hence by $C$. Since $\lambda^{\infty}(x)-\lambda^{\infty}(0)$ is bounded on $\Omega$ by $C|x|$, the third term is bounded by $C\left\||z| \int_{V} \tilde{u}^{\varepsilon}\right\|+\sqrt{\varepsilon} C\left\|\tilde{u}^{\varepsilon}\right\|$ and hence by $C$. This concludes the proof of the lemma.

We now extend $\tilde{u}^{\varepsilon}$ to $\mathbb{R}^{N}$ as in the preceding section by imposing that it solve (47), that it be continuous across the interface $\partial \Omega^{\varepsilon} \times V$, and that $\tilde{u}^{\varepsilon}(x, v) \rightarrow 0$ as $|x| \rightarrow \infty$. The very strong absorption in $\mathbb{R}^{N} \backslash \Omega^{\varepsilon}$ allows us to prove by integration along characteristics that the above a priori estimates also hold for the extended function $\tilde{u}^{\varepsilon}$ (remark that we need the estimates on $\Gamma_{\varepsilon}^{+}$to establish the following corollary). Thus we obtained

Corollary 4.2. Let $\tilde{u}^{\varepsilon}$ be defined on $\mathbb{R}^{N} \times V$ as above. Then we have

$$
\begin{aligned}
\left\|\tilde{u}^{\varepsilon}\right\|_{L^{2}\left(\mathbb{R}^{N} \times V\right)}+\left\|v \cdot \nabla \tilde{u}^{\varepsilon}\right\|_{L^{2}\left(\mathbb{R}^{N} \times V\right)}+\left\||z| \int_{V} \tilde{u}^{\varepsilon}\right\|_{L^{2}\left(\mathbb{R}^{N} \times V\right)} & \\
+\frac{1}{\sqrt{\varepsilon}}\left\|\tilde{u}^{\varepsilon}-\int_{V} \tilde{u}^{\varepsilon}\right\|_{L^{2}\left(\mathbb{R}^{N} \times V\right)} & \leq C\|\tilde{q}\|_{L^{2}\left(\Omega^{\varepsilon} \times V\right)} .
\end{aligned}
$$

We conclude this section by stating an important result, which derives from the above a priori estimates,

Corollary 4.3. Let $\tilde{q}^{\varepsilon}$ be a bounded sequence of $L^{2}\left(\mathbb{R}^{N} \times V\right)$. Let $\tilde{u}^{\varepsilon}$ be the solution of (46), where $\tilde{q}$ is replaced by $\tilde{q}^{\varepsilon}$, and then extended to $\mathbb{R}^{N} \times V$ as above. Then, the sequence $\tilde{u}^{\varepsilon}$ is relatively compact in $L^{2}\left(\mathbb{R}^{N} \times V\right)$.

Proof. The previous a priori estimates still hold when $\tilde{q}$ is replaced by $\tilde{q}^{\varepsilon}$. Therefore, there exists $C>0$ such that

$$
\left\|\tilde{u}^{\varepsilon}\right\|_{L^{2}\left(\mathbb{R}^{N} \times V\right)}+\left\|v \cdot \nabla \tilde{u}^{\varepsilon}\right\|_{L^{2}\left(\mathbb{R}^{N} \times V\right)}+\left\||z| \int_{V} \tilde{u}^{\varepsilon}\right\|_{L^{2}\left(\mathbb{R}^{N} \times V\right)} \leq C .
$$

Using Lemma 1.8, we deduce that the sequence $\int_{V} \tilde{u}^{\varepsilon}$ is relatively compact. But we also know that $\left\|\tilde{u}^{\varepsilon}-\int_{V} \tilde{u}^{\varepsilon}\right\|_{L^{2}\left(\mathbb{R}^{N} \times V\right)} \leq C \sqrt{\varepsilon}$. This proves the relative compactness of the sequence $\tilde{u}^{\varepsilon}$.

\section{Convergence proof}

The aim of this section is to prove Theorem 3.6. It is based on the use of the two-scale convergence technique $[1,2]$.

We first introduce some notation and denote by $\mathcal{C}_{\#}^{\infty}(Y)$ the space of infinitely differentiable functions in $\mathbb{R}^{N}$ that are $Y$-periodic, and $L_{\#}^{2}(Y)$ (respectively $\left.H_{\#}^{1}(Y)\right)$ the completion of $\mathcal{C}_{\#}^{\infty}(Y)$ for the norm of $L_{\#}^{2}(Y)$ (respectively of $H_{\#}^{1}(Y)$ ). Since our functions oscillate with period $\sqrt{\varepsilon}$ on $\mathbb{R}^{N}$, our definition of two-scale convergence is here: 
Definition 5.1. A sequence of functions $g^{\varepsilon}$ in $L^{2}\left(\mathbb{R}^{N} \times V\right)$ is said to two-scale converge to a limit $g$ in $L^{2}\left(\mathbb{R}^{N}\right.$ $\times Y \times V)$ if, for any function $\psi$ in $\mathcal{D}\left(\mathbb{R}^{N} \times V ; \mathcal{C}_{\#}^{\infty}(Y)\right)$, we have

$$
\lim _{\varepsilon \rightarrow 0} \int_{\mathbb{R}^{N}} \int_{V} g^{\varepsilon}(x, v) \psi\left(x, \frac{x}{\sqrt{\varepsilon}}, v\right) \mathrm{d} x \mathrm{~d} v=\int_{\mathbb{R}^{N}} \int_{Y} \int_{V} g(x, y, v) \psi(x, y, v) \mathrm{d} x \mathrm{~d} y \mathrm{~d} v .
$$

We also recall here an important result of two-scale convergence:

Theorem 5.2. Let $g^{\varepsilon}$ be a bounded sequence in $L^{2}\left(\mathbb{R}^{N} \times V\right)$. Then there exists a limit $g$ in $L^{2}\left(\mathbb{R}^{N} \times Y \times V\right)$ such that, up to a subsequence, $g^{\varepsilon}$ two-scale converges to $g$.

The a priori estimates obtained for $\tilde{u}^{\varepsilon}$ will allow us to prove a result of two-scale convergence for $\tilde{u}^{\varepsilon}$, and to guess what form its limit should have. This is the goal of the next proposition:

Proposition 5.3. Let $\tilde{u}^{\varepsilon}$ be a sequence in $L^{2}\left(\mathbb{R}^{N} \times V\right)$ such that there exists a constant $C$ independent of $\varepsilon$ satisfying the following energy estimate

$$
\left\|\tilde{u}^{\varepsilon}\right\|_{L^{2}\left(\mathbb{R}^{N} \times V\right)}+\left\|v \cdot \nabla \tilde{u}^{\varepsilon}\right\|_{L^{2}\left(\mathbb{R}^{N} \times V\right)}+\frac{1}{\sqrt{\varepsilon}}\left\|\tilde{u}^{\varepsilon}-\int_{V} \tilde{u}^{\varepsilon}\right\|_{L^{2}\left(\mathbb{R}^{N} \times V\right)}+\left\||z| \int_{V} \tilde{u}^{\varepsilon}\right\|_{L^{2}\left(\mathbb{R}^{N} \times V\right)} \leq C .
$$

Then, there exists $u^{0}(z)$ in $H^{1}\left(\mathbb{R}^{N}\right) \cap L_{z}^{2}\left(\mathbb{R}^{N}\right)$ and $u^{1}(z, y)$ in $L^{2}\left(\mathbb{R}^{N} \times V ; H_{\#}^{1}(Y)\right)$ such that, up to a subsequence, $\tilde{u}^{\varepsilon}(z, v)$ strongly converges to $u^{0}(z)$ in $L^{2}\left(\mathbb{R}^{N}\right), v \cdot \nabla \tilde{u}^{\varepsilon}$ two-scale converges to $v \cdot \nabla_{z} u^{0}+v \cdot \nabla_{y} u^{1}$, and $\frac{1}{\sqrt{\varepsilon}}\left(\tilde{u}^{\varepsilon}\right.$ $\left.-\int_{V} \tilde{u}^{\varepsilon}\right)$ two-scale converges to $u^{1}-\int_{V} u^{1}$.

The proof of this proposition follows from minor modifications of that of [2] (Prop. 5.3). We deduce the following result from the above proposition.

Proposition 5.4. Assume that hypotheses (H1-H4) hold. Let $\tilde{u}^{\varepsilon}$ be the unique solution to (46) extended to $\mathbb{R}^{N}$ by imposing that it solve (47), that it be continuous across the interface $\partial \Omega^{\varepsilon} \times V$, and that $\tilde{u}^{\varepsilon}(x, v) \rightarrow 0$ as $|x| \rightarrow \infty$. With the notation of Proposition 5.3, $u^{1}$ is given by

$$
u^{1}(z, y, v)=\sum_{j=1}^{N} \frac{\partial u^{0}}{\partial z_{j}}(z) \theta^{j}(y, v),
$$

where $\theta^{j}(y, v)$ is the unique solution of

$$
\left\{\begin{array}{l}
v \cdot \nabla_{y} \theta^{j}+Q\left(0, \theta^{j}\right)=-v_{j} \quad \text { in } Y \times V, \\
y \mapsto \theta^{j}(y, v) Y-\text { periodic },
\end{array}\right.
$$

up to an additive constant. The operator $Q(x, \cdot)$ is defined by

$$
\begin{aligned}
Q(x, u)(y, v)= & \frac{u(y, v)}{\psi(x, y, v)} \int_{V} \sigma_{\infty}\left(x, y, v^{\prime}, v\right) \psi\left(x, y, v^{\prime}\right) \mathrm{d} v^{\prime} \\
& -\frac{1}{\psi(x, y, v)} \int_{V} \sigma_{\infty}\left(x, y, v^{\prime}, v\right) \psi\left(x, y, v^{\prime}\right) u\left(y, v^{\prime}\right) \mathrm{d} v^{\prime}
\end{aligned}
$$

and the adjoint operator $Q^{*}(x, \cdot)$ by

$$
\begin{aligned}
Q^{*}(x, u)(y, v)= & \frac{u(y, v)}{\psi(x, y, v)} \int_{V} \sigma_{\infty}\left(x, y, v^{\prime}, v\right) \psi\left(x, y, v^{\prime}\right) \mathrm{d} v^{\prime} \\
& -\psi(x, y, v) \int_{V} \sigma_{\infty}^{*}\left(x, y, v^{\prime}, v\right) \frac{1}{\psi(x, y, v)} u\left(y, v^{\prime}\right) \mathrm{d} v^{\prime} .
\end{aligned}
$$


Proof. Let $\phi(x, y, v)$ be a smooth $Y$-periodic function with compact support in its first variable. Multiplying (46) by $\sqrt{\varepsilon} \phi\left(z, \frac{z}{\sqrt{\varepsilon}}, v\right)$ and integrating over $\mathbb{R}^{N} \times V$ yields

$$
\int_{\mathbb{R}^{N}} \int_{V} v \cdot \nabla \tilde{u}^{\varepsilon} \phi \mathrm{d} z \mathrm{~d} v+\int_{\mathbb{R}^{N}} \int_{V} \tilde{Q}^{\varepsilon}\left(\tilde{w}^{\varepsilon}\right) \phi \mathrm{d} z \mathrm{~d} v=\sqrt{\varepsilon} \int_{\mathbb{R}^{N}} \int_{V} \tilde{S}^{\varepsilon} \phi, \mathrm{d} z \mathrm{~d} v,
$$

where $\tilde{w}^{\varepsilon}=\frac{\tilde{u}^{\varepsilon}-\int_{V} \tilde{u}^{\varepsilon}}{\sqrt{\varepsilon}}$, and where the source term is

$$
\tilde{S}^{\varepsilon}=-\tilde{\alpha}^{\varepsilon} \tilde{u}^{\varepsilon}-\left(\frac{\lambda^{\infty}(\sqrt{\varepsilon} z)-\lambda^{\infty}(0)}{\varepsilon}+\eta\right) \tilde{F}^{\varepsilon}\left(\tilde{u}^{\varepsilon}\right)+\tilde{F}^{\varepsilon}(\tilde{q}) .
$$

The difference $\frac{\lambda^{\infty}(\sqrt{\varepsilon} z)-\lambda^{\infty}(0)}{\sqrt{\varepsilon}}$ is clearly bounded in $\Omega^{\varepsilon}$ by $C \sqrt{\varepsilon} z \cdot z$. Thus, since $\phi$ has compact support, the right-hand side in (62) converges to 0 as $\varepsilon$ goes to 0 . The first term in (62) converges to $\int_{\mathbb{R}^{N}} \int_{Y} \int_{V}\left(v \cdot \nabla_{z} u^{0}\right.$ $\left.+v \cdot \nabla_{y} u^{1}\right) \phi \mathrm{d} z \mathrm{~d} y \mathrm{~d} v$ as $\varepsilon$ goes to 0 .

To study the convergence of the second term, we need to introduce some notation. We define

$$
\tilde{\psi}_{0}^{\varepsilon}(z, v)=\psi\left(0, \frac{z}{\sqrt{\varepsilon}}, v\right)
$$

and similarly $\tilde{\psi}_{0}^{* \varepsilon}, \tilde{\sigma}_{\infty 0}^{\varepsilon}, f_{0}^{\varepsilon}$, and the operators $\tilde{Q}_{0}^{\varepsilon}$ and $\tilde{F}_{0}^{\varepsilon}$. By Lipschitz regularity of all physical parameters, we deduce that

$$
\left|\int_{\mathbb{R}^{N}} \int_{V}\left(\tilde{Q}^{\varepsilon}-\tilde{Q}_{0}^{\varepsilon}\right)\left(\tilde{w}^{\varepsilon}\right) \phi \mathrm{d} z \mathrm{~d} v\right| \leq C(\phi) \sqrt{\varepsilon}\left\|\tilde{w}^{\varepsilon}\right\|_{L^{2}\left(\mathbb{R}^{N} \times V\right)} .
$$

Introducing the adjoint scattering kernel $\tilde{Q}_{0}^{* \varepsilon}$, we obtain

$$
\int_{\mathbb{R}^{N}} \int_{V} \tilde{Q}^{\varepsilon}\left(\tilde{w}^{\varepsilon}\right) \phi \mathrm{d} z \mathrm{~d} v=\int_{\mathbb{R}^{N}} \int_{V} \tilde{w}^{\varepsilon} \tilde{Q}_{0}^{* \varepsilon}(\phi) \mathrm{d} z \mathrm{~d} v+O(\sqrt{\varepsilon})
$$

Next we check that $\tilde{Q}_{0}^{* \varepsilon}\left(\phi\left(z, \frac{z}{\sqrt{\varepsilon}}, v\right)\right)$ two-scale converges to $Q^{*}(0, \phi)(z, y, v)$ and that

$$
\lim _{\varepsilon \rightarrow 0}\left\|\tilde{Q}_{0}^{* \varepsilon}\left(\phi\left(z, \frac{z}{\sqrt{\varepsilon}}, v\right)\right)\right\|_{L 2\left(\mathbb{R}^{N} \times V\right)}=\left\|Q^{*}(0, \phi)(z, y, v)\right\|_{L 2\left(\mathbb{R}^{N} \times V\right)} .
$$

This last property allows us to pass to the limit in a product of two weakly converging sequences [1]. Since $w^{\varepsilon}$ two-scale converges to $u_{\varepsilon}-\int_{V} u_{\varepsilon}$, we get in the limit that

$$
\int_{\mathbb{R}^{N}} \int_{Y} \int_{V}\left(v \cdot \nabla_{z} u^{0}+v \cdot \nabla_{y} u^{1}\right) \phi \mathrm{d} z \mathrm{~d} y \mathrm{~d} v+\int_{\mathbb{R}^{N}} \int_{Y} \int_{V} Q\left(0, u^{1}-\int_{V} u^{1}\right) \phi \mathrm{d} z \mathrm{~d} y \mathrm{~d} v=0 .
$$

Thus $u^{1}$ is a solution of the following equation

$$
\left\{\begin{array}{l}
v \cdot \nabla_{y} u^{1}+Q\left(0, u^{1}\right)=-v \cdot \nabla_{z} u^{o} \\
y \mapsto u^{1}(z, y, v) Y-\text { periodic. }
\end{array}\right.
$$

Since $u^{0}$ depends only on $z$, we deduce that $u^{1}(z, y, v)=\sum_{j=1}^{N} \frac{\partial u^{0}}{\partial z_{j}}(z) \theta^{j}(y, v)$, where $\theta^{j}$ is a solution of the following equation

$$
\left\{\begin{array}{l}
v \cdot \nabla_{y} \theta^{j}+Q\left(0, \theta^{j}\right)=-v_{j} \\
y \mapsto \theta^{j}(y, v) Y-\text { periodic. }
\end{array}\right.
$$


It is easy to see that $\chi^{j}=\theta^{j} \psi^{0}$, and therefore, thanks to hypothesis (H4), this last equation is solvable, and has a unique solution, up to an additive constant. This concludes the proof of our proposition.

Proposition 5.5. With the same hypotheses as in the previous proposition, the sequence $\tilde{u}^{\varepsilon}(z, v)$ converges strongly in $L^{2}\left(\mathbb{R}^{N} \times V\right)$ to $u^{0}(z) \in H^{1}\left(\mathbb{R}^{N}\right)$, solution of the following problem

$$
\left\{\begin{array}{l}
-\operatorname{div}\left(\bar{D} \nabla u^{0}\right)+(\bar{A} z . z+\bar{\gamma}+\eta \bar{\sigma}) u^{0}+\left(\operatorname{div}\left(\bar{B}^{*} z u^{0}\right)-\operatorname{div}(J)(0) u^{0}\right)=\overline{F(\tilde{q})} \\
u^{0} \in H^{1}\left(\mathbb{R}^{N}\right) \cap L_{z}^{2}\left(\mathbb{R}^{N}\right)
\end{array}\right.
$$

where the coefficients are defined in (34) and (49).

Proof. Let us first define, for $1 \leq i \leq n$, the adjoint cell problem at $x=0$

$$
\left\{\begin{array}{l}
-v \cdot \nabla_{y}\left(\psi \psi^{*} \theta^{* i}\right)+Q^{*}\left(0, \psi \psi^{*} \theta^{* i}\right)=v_{i} \psi \psi^{*} \\
y \mapsto \theta^{* i}(y, v) Y \text { - periodic }
\end{array}\right.
$$

which admits a unique solution, up to an additive constant, since $\int_{Y} \int_{V} v_{i} \psi \psi^{*} \mathrm{~d} y \mathrm{~d} v=0$ at $x=0$. Let $\phi(z)$ be a smooth function with compact support in $\mathbb{R}^{N}$. We define

$$
\phi^{\varepsilon}(z, v)=\phi(z)+\sqrt{\varepsilon} \sum_{j=1}^{N} \frac{\partial \phi}{\partial z_{j}}(z) \theta^{* j}\left(\frac{z}{\sqrt{\varepsilon}}, v\right)
$$

Upon multiplying (46) by $\phi^{\varepsilon} \tilde{\psi}^{\varepsilon} \tilde{\psi}^{* \varepsilon}$ and integrating over $\mathbb{R}^{N} \times V$, we obtain

$$
\begin{aligned}
& \int_{\mathbb{R}^{N}} \int_{V} \frac{1}{\sqrt{\varepsilon}} v \cdot \nabla \tilde{u}^{\varepsilon} \phi^{\varepsilon} \tilde{\psi}^{\varepsilon} \tilde{\psi}^{* \varepsilon}+\int_{\mathbb{R}^{N}} \int_{V} \tilde{\alpha}^{\varepsilon} \tilde{u}^{\varepsilon} \phi^{\varepsilon} \tilde{\psi}^{\varepsilon} \tilde{\psi}^{* \varepsilon}+\int_{\mathbb{R}^{N}} \int_{V} \frac{1}{\varepsilon} \tilde{Q}^{\varepsilon}\left(\tilde{u}^{\varepsilon}\right) \phi^{\varepsilon} \tilde{\psi}^{\varepsilon} \tilde{\psi}^{* \varepsilon} \\
& +\int_{\mathbb{R}^{N}} \int_{V}\left(\frac{\lambda^{\infty}(\sqrt{\varepsilon} z)-\lambda^{\infty}(0)}{\varepsilon}+\eta\right) \tilde{F}^{\varepsilon}\left(\tilde{u}^{\varepsilon}\right) \phi^{\varepsilon} \tilde{\psi}^{\varepsilon} \tilde{\psi}^{* \varepsilon}=\int_{\mathbb{R}^{N}} \int_{V} \tilde{F}^{\varepsilon}(\tilde{q}) \phi^{\varepsilon} \tilde{\psi}^{\varepsilon} \tilde{\psi}^{* \varepsilon}
\end{aligned}
$$

Denoting these integrals by $J_{i}^{\varepsilon}$ in the same order, (65) reads

$$
J_{1}^{\varepsilon}+J_{2}^{\varepsilon}+J_{3}^{\varepsilon}+J_{4}^{\varepsilon}=J_{5}^{\varepsilon} .
$$

We now pass to the limit in each term $J_{i}^{\varepsilon}$. The right-hand side is given by

$$
\begin{gathered}
J_{5}^{\varepsilon}=\int_{\mathbb{R}^{N}} \int_{V} \int_{V} f\left(\sqrt{\varepsilon} z, \frac{z}{\sqrt{\varepsilon}}, v^{\prime}, v\right) \psi\left(\sqrt{\varepsilon} z, \frac{z}{\sqrt{\varepsilon}}, v^{\prime}\right) \tilde{q}\left(z, v^{\prime}\right) \psi^{*}\left(\sqrt{\varepsilon} z, \frac{z}{\sqrt{\varepsilon}}, v\right) . \\
\cdot\left[\phi(z)+\sqrt{\varepsilon} \sum_{j=1}^{N} \frac{\partial \phi}{\partial z_{j}} \theta^{* j}\left(\frac{z}{\sqrt{\varepsilon}}, v\right)\right] \mathrm{d} z \mathrm{~d} v \mathrm{~d} v^{\prime} .
\end{gathered}
$$

By Lipschitz regularity of the functions $f, \psi$, and $\psi^{*}$, we have

$$
J_{5}^{\varepsilon}=\int_{\mathbb{R}^{N}} \int_{V} \int_{V} f\left(0, \frac{z}{\sqrt{\varepsilon}}, v^{\prime}, v\right) \psi\left(0, \frac{z}{\sqrt{\varepsilon}}, v^{\prime}\right) \tilde{q}\left(z, v^{\prime}\right) \psi^{*}\left(0, \frac{z}{\sqrt{\varepsilon}}, v\right) \phi(z) \mathrm{d} z \mathrm{~d} v \mathrm{~d} v^{\prime}+C(\phi) \sqrt{\varepsilon} .
$$

Thus, it converges to

$$
J_{5}=\int_{\mathbb{R}^{N}} \int_{Y} \int_{V} \int_{V} f\left(0, y, v^{\prime}, v\right) \psi\left(0, y, v^{\prime}\right) \tilde{q}\left(z, v^{\prime}\right) \psi^{*}(0, y, v) \phi(z) \mathrm{d} z \mathrm{~d} v \mathrm{~d} v^{\prime} .
$$


The fourth term is

$$
\begin{aligned}
J_{4}^{\varepsilon}= & \int_{\mathbb{R}^{N}} \int_{V} \int_{V}\left(\frac{\lambda^{\infty}(\sqrt{\varepsilon} z)-\lambda^{\infty}(0)}{\varepsilon}+\eta\right) f\left(\sqrt{\varepsilon} z, \frac{z}{\sqrt{\varepsilon}}, v^{\prime}, v\right) \psi\left(\sqrt{\varepsilon} z, \frac{z}{\sqrt{\varepsilon}}, v^{\prime}\right) \\
& \times \tilde{u}^{\varepsilon}\left(z, v^{\prime}\right) \phi(z) \psi^{*}\left(\sqrt{\varepsilon} z, \frac{z}{\sqrt{\varepsilon}}, v\right) \mathrm{d} z \mathrm{~d} v \mathrm{~d} v^{\prime} \\
& +\sqrt{\varepsilon} \int_{\mathbb{R}^{N}} \int_{V} \int_{V}\left(\frac{\lambda^{\infty}(\sqrt{\varepsilon} z)-\lambda^{\infty}(0)}{\varepsilon}+\eta\right) f\left(\sqrt{\varepsilon} z, \frac{z}{\sqrt{\varepsilon}}, v^{\prime}, v\right) \\
& \times \psi\left(\sqrt{\varepsilon} z, \frac{z}{\sqrt{\varepsilon}}, v^{\prime}\right) \tilde{u}^{\varepsilon}\left(z, v^{\prime}\right) \sum_{j=1}^{N} \frac{\partial \phi}{\partial z_{j}} \theta^{* j} \psi^{*}\left(\sqrt{\varepsilon} z, \frac{z}{\sqrt{\varepsilon}}, v\right) \mathrm{d} z \mathrm{~d} v \mathrm{~d} v^{\prime},
\end{aligned}
$$

or, by Lipschitz regularity,

$$
\begin{aligned}
J_{4}^{\varepsilon}= & \int_{\mathbb{R}^{N}} \int_{V} \int_{V}\left(\frac{\lambda^{\infty}(\sqrt{\varepsilon} z)-\lambda^{\infty}(0)}{\varepsilon}+\eta\right) \tilde{u}^{\varepsilon}\left(z, v^{\prime}\right) \phi(z) f \\
& \times\left(0, \frac{z}{\sqrt{\varepsilon}}, v^{\prime}, v\right) \psi\left(0, \frac{z}{\sqrt{\varepsilon}}, v^{\prime}\right) \psi^{*}\left(0, \frac{z}{\sqrt{\varepsilon}}, v\right) \mathrm{d} z \mathrm{~d} v \mathrm{~d} v^{\prime}+O(\sqrt{\varepsilon})
\end{aligned}
$$

and, thus, converges to

$$
J_{4}=\int_{\mathbb{R}^{N}} \int_{Y} \int_{V} \int_{V}\left(\lambda_{i j}^{2} z_{i} z_{j}+\eta\right) f\left(0, y, v^{\prime}, v\right) \psi\left(0, y, v^{\prime}\right) \psi^{*}(0, y, v) u_{0}(z) \phi(z) \mathrm{d} z \mathrm{~d} y \mathrm{~d} v \mathrm{~d} v^{\prime}
$$

The second term is

$$
\begin{aligned}
J_{2}^{\varepsilon}= & \int_{\mathbb{R}^{N}} \int_{V} v \cdot \frac{\nabla_{x} \psi}{\psi}\left(\sqrt{\varepsilon} z, \frac{z}{\sqrt{\varepsilon}}, v\right) \tilde{u}^{\varepsilon}(z, v) \phi(z)\left(\psi \psi^{*}\right)\left(\sqrt{\varepsilon} z, \frac{z}{\sqrt{\varepsilon}}, v\right) \mathrm{d} z \mathrm{~d} v \mathrm{~d} v^{\prime} \\
& +\sqrt{\varepsilon} \int_{\mathbb{R}^{N}} \int_{V} v \cdot \frac{\nabla_{x} \psi}{\psi}\left(\sqrt{\varepsilon} z, \frac{z}{\sqrt{\varepsilon}}, v\right) \tilde{u}^{\varepsilon}(z, v) \sum_{j=1}^{N} \frac{\partial \phi}{\partial z_{j}} \theta^{* j}\left(\frac{z}{\sqrt{\varepsilon}}, v\right)\left(\psi \psi^{*}\right)\left(\sqrt{\varepsilon} z, \frac{z}{\sqrt{\varepsilon}}, v\right) \mathrm{d} z \mathrm{~d} v \mathrm{~d} v^{\prime}
\end{aligned}
$$

or, by Lipschitz regularity,

$$
J_{2}^{\varepsilon}=\int_{\mathbb{R}^{N}} \int_{V} v \cdot \frac{\nabla_{x} \psi}{\psi}\left(0, \frac{z}{\sqrt{\varepsilon}}, v\right) \tilde{u}^{\varepsilon}(z, v) \phi(z)\left(\psi \psi^{*}\right)\left(0, \frac{z}{\sqrt{\varepsilon}}, v\right) \mathrm{d} z \mathrm{~d} v \mathrm{~d} v^{\prime}+O(\sqrt{\varepsilon})
$$

and thus converges to

$$
J_{2}=\int_{\mathbb{R}^{N}} \int_{Y} \int_{V} v \cdot \nabla_{x} \psi(0, y, v) \psi^{*}(0, y, v) u_{0}(z) \phi(z) \mathrm{d} z \mathrm{~d} y \mathrm{~d} v
$$


Let us next deal with the sum $J_{1}^{\varepsilon}+J_{3}^{\varepsilon}$. After integrating by parts, $J_{1}^{\varepsilon}$ can be written as the sum of seven integrals

$$
\left\{\begin{array}{l}
K_{1}^{\varepsilon}=-\frac{1}{\sqrt{\varepsilon}} \int_{\mathbb{R}^{N}} \int_{V} \tilde{u}^{\varepsilon} v \cdot \nabla_{z} \phi \tilde{\psi}^{\varepsilon} \tilde{\psi}^{* \varepsilon} \\
K_{2}^{\varepsilon}=-\int_{\mathbb{R}^{N}} \int_{V} \tilde{u}^{\varepsilon} v \cdot \sum_{j=1}^{N} \nabla_{z} \frac{\partial \phi}{\partial z_{j}} \theta^{* j} \tilde{\psi}^{\varepsilon} \tilde{\psi}^{* \varepsilon} \\
K_{3}^{\varepsilon}=-\frac{1}{\sqrt{\varepsilon}} \int_{\mathbb{R}^{N}} \int_{V} \tilde{u}^{\varepsilon} v \cdot \sum_{j=1}^{N} \frac{\partial \phi}{\partial z_{j}} \nabla_{y} \theta^{* j} \tilde{\psi}^{\varepsilon} \tilde{\psi}^{* \varepsilon} \\
K_{4}^{\varepsilon}=-\int_{\mathbb{R}^{N}} \int_{V} \tilde{u}^{\varepsilon} v \cdot \phi\left(\widetilde{\nabla_{x} \psi \psi^{*}}\right)^{\varepsilon} \\
K_{5}^{\varepsilon}=-\frac{1}{\varepsilon} \int_{\mathbb{R}^{N}} \int_{V} \tilde{u}^{\varepsilon} v \cdot \phi\left(\widetilde{\nabla_{y} \psi \psi^{*}}\right)^{\varepsilon} \\
K_{6}^{\varepsilon}=-\sqrt{\varepsilon} \int_{\mathbb{R}^{N}} \int_{V} \tilde{u}^{\varepsilon} v \cdot \sum_{j=1}^{N} \frac{\partial \phi}{\partial z_{j}} \theta^{* j}\left(\widetilde{\nabla_{x} \psi \psi^{*}}\right)^{\varepsilon} \\
K_{7}^{\varepsilon}=-\frac{1}{\sqrt{\varepsilon}} \int_{\mathbb{R}^{N}} \int_{V} \tilde{u}^{\varepsilon} v \cdot \sum_{j=1}^{N} \frac{\partial \phi}{\partial z_{j}} \theta^{* j}\left(\widetilde{\nabla_{y} \psi \psi^{*}}\right)^{\varepsilon}
\end{array}\right.
$$

and $J_{3}^{\varepsilon}$ is the sum of two integrals

$$
\left\{\begin{aligned}
K_{8}^{\varepsilon} & =\frac{1}{\varepsilon} \int_{\mathbb{R}^{N}} \int_{V} \tilde{Q}^{\varepsilon}\left(\tilde{u}^{\varepsilon}\right) \phi \tilde{\psi}^{\varepsilon} \tilde{\psi}^{* \varepsilon} \\
K_{9}^{\varepsilon} & =\frac{1}{\sqrt{\varepsilon}} \int_{\mathbb{R}^{N}} \int_{V} \tilde{Q}^{\varepsilon}\left(\tilde{u}^{\varepsilon}\right) \sum_{j=1}^{N} \frac{\partial \phi}{\partial z_{j}} \theta^{* j} \tilde{\psi}^{\varepsilon} \tilde{\psi}^{* \varepsilon}
\end{aligned}\right.
$$

We first observe that $K_{6}^{\varepsilon}$ converges to 0 . Now, recalling that

$$
-v \cdot \nabla_{y}\left(\psi \psi^{*}\right)+Q^{*}\left(\psi \psi^{*}\right)=0
$$

for each $x \in \Omega$, we have

$$
K_{5}^{\varepsilon}+K_{8}^{\varepsilon}=0 .
$$

Again, we use Lipschitz regularity to show that $K_{2}^{\varepsilon}$ and $K_{4}^{\varepsilon}$ converge to

$$
K_{2}=-\int_{\mathbb{R}^{N}} \int_{Y} \int_{V} v_{i} \theta^{* j}(y, v) \psi(0, y, v) \psi^{*}(0, y, v) u^{0}(z) \frac{\partial^{2} \phi}{\partial z_{i} z_{j}}(z) \mathrm{d} z \mathrm{~d} y \mathrm{~d} v
$$

and

$$
\begin{aligned}
K_{4} & =-\int_{\mathbb{R}^{N}} \int_{Y} \int_{V} v \cdot \nabla_{x}\left(\psi \psi^{*}\right)(0, y, v) u^{0}(z) \phi(z) \mathrm{d} z \mathrm{~d} y \mathrm{~d} v \\
& =-\int_{\mathbb{R}^{N}} \operatorname{div}(J)(0) u^{0}(z) \phi(z) \mathrm{d} z \mathrm{~d} y \mathrm{~d} v
\end{aligned}
$$

respectively. Since we assume that our data are of class $\mathcal{C}^{2}$ with respect to the slow variable $x$, we have for instance

$$
\left\{\begin{array}{l}
\left.\widetilde{\left(\psi \psi^{*}\right.}\right)^{\varepsilon}(z, v)=\psi \psi^{*}\left(0, \frac{z}{\sqrt{\varepsilon}}, v\right)+\sqrt{\varepsilon} z \cdot \nabla_{x}\left(\psi \psi^{*}\right)\left(0, \frac{z}{\sqrt{\varepsilon}}, v\right)+O(\varepsilon) \\
\tilde{Q}^{* \varepsilon}(h)(z, v)=Q^{*}(0, h)\left(0, \frac{z}{\sqrt{\varepsilon}}, v\right)+\sqrt{\varepsilon} z \cdot \nabla_{x} Q^{*}(0, h)\left(0, \frac{z}{\sqrt{\varepsilon}}, v\right)+O(\varepsilon) .
\end{array}\right.
$$


Therefore, the sum $K_{1}^{\varepsilon}+K_{3}^{\varepsilon}+K_{7}^{\varepsilon}+K_{9}^{\varepsilon}$ is equal to $\frac{S_{1}^{\varepsilon}}{\sqrt{\varepsilon}}+S_{2}^{\varepsilon}+O(\sqrt{\varepsilon})$, where

$$
\begin{aligned}
S_{1}^{\varepsilon}=\int_{\mathbb{R}^{N}} \int_{V} & -\tilde{u}^{\varepsilon}(z, v) v \cdot\left(\nabla_{z} \phi\right)(z)\left(\psi \psi^{*}\right)\left(0, \frac{z}{\sqrt{\varepsilon}}, v\right) \\
& -\tilde{u}^{\varepsilon}(z, v) v \cdot \sum_{j=1}^{N} \frac{\partial \phi}{\partial z}\left(z_{j}\right) \nabla_{y}\left(\theta^{* j} \psi \psi^{*}\right)\left(0, \frac{z}{\sqrt{\varepsilon}}, v\right) \\
& +\tilde{u}^{\varepsilon}(z, v) \sum_{j=1}^{N} \frac{\partial \phi}{\partial z_{j}} Q^{*}\left(0, \theta^{* j} \psi \psi^{*}\right)\left(\frac{z}{\sqrt{\varepsilon}}, v\right),
\end{aligned}
$$

and

$$
\begin{aligned}
S_{2}^{\varepsilon}=\int_{\mathbb{R}^{N}} \int_{V} & -\tilde{u}^{\varepsilon}(z, v) v \cdot\left(\nabla_{z} \phi\right)(z) z \cdot \nabla_{x}\left(\psi \psi^{*}\right)\left(0, \frac{z}{\sqrt{\varepsilon}}, v\right) \\
& -\tilde{u}^{\varepsilon}(z, v) \sum_{j=1}^{N} \frac{\partial \phi}{\partial z_{j}}(z) v \cdot \nabla_{y}\left(\theta^{* j} z \cdot \nabla_{x}\left(\psi \psi^{*}\right)\right)\left(0, \frac{z}{\sqrt{\varepsilon}}, v\right) \\
& +\tilde{u}^{\varepsilon}(z, v) \sum_{j=1}^{N} \frac{\partial \phi}{\partial z_{j}} z \cdot \nabla_{x}\left(Q^{*}\right)\left(0, \theta^{* j} \psi \psi^{*}\right)\left(\frac{z}{\sqrt{\varepsilon}}, v\right) \\
& +\tilde{u}^{\varepsilon}(z, v) \sum_{j=1}^{N} \frac{\partial \phi}{\partial z_{j}} Q^{*}\left(0, \theta^{* j} z \cdot \nabla_{x}\left(\psi \psi^{*}\right)\left(\frac{z}{\sqrt{\varepsilon}}, v\right)\right.
\end{aligned}
$$

Recalling that $\theta^{* j}$ is the solution of the adjoint cell problem (64)

$$
-v \cdot \nabla_{y}\left(\psi \psi^{*} \theta^{* j}\right)+Q^{*}\left(\psi \psi^{*} \theta^{* j}\right)=v_{j} \psi \psi^{*}
$$

we obtain that $S_{1}^{\varepsilon}=0$. Next, $S_{2}^{\varepsilon}$ converges to

$$
\begin{aligned}
S_{2}=\int_{\mathbb{R}^{N}} \int_{Y} \int_{V} & -v_{i} \frac{\partial\left(\psi \psi^{*}\right)}{\partial x_{j}}(0, y, v) \frac{\partial \phi}{\partial z_{i}} z_{j} u^{0}(z) \\
& -u^{0}(z) \sum_{j=1}^{N} \frac{\partial \phi}{\partial z_{j}}(z) v \cdot \nabla_{y}\left(\theta^{* j} z \cdot \nabla_{x}\left(\psi \psi^{*}\right)\right)(0, y, v) \\
& +u^{0}(z) \sum_{j=1}^{N} \frac{\partial \phi}{\partial z_{j}}(z) z \cdot \nabla_{x}\left(Q^{*}\right)\left(0, \theta^{* j} \psi \psi^{*}\right)(y, v) \\
& +u^{0}(z) \sum_{j=1}^{N} \frac{\partial \phi}{\partial z_{j}}(z) Q^{*}\left(0, \theta^{* j} z \cdot \nabla_{x}\left(\psi \psi^{*}\right)\right)(y, v) \mathrm{d} z \mathrm{~d} y \mathrm{~d} v
\end{aligned}
$$

It is straightforward to verify that the last three terms in $S_{2}$ vanish. After integrating by parts, we have

$$
S_{2}=\int_{\mathbb{R}^{N}} \int_{Y} \int_{V} \partial_{z_{i}}\left(v_{i} \partial_{x_{j}}\left(\psi \psi^{*}\right) z_{j} u^{0}\right) \phi^{0} \mathrm{~d} z \mathrm{~d} y \mathrm{~d} v=\int_{\mathbb{R}^{N}} \operatorname{div}\left(\bar{B}^{*} z u^{0}\right) \phi^{0} \mathrm{~d} z
$$

Eventually, passing to the limit yields

$$
K_{2}+J_{2}+J_{4}+S_{2}+K_{4}=J_{5}
$$


or equivalently,

$$
\begin{aligned}
& -\int_{\mathbb{R}^{N}} \int_{Y} \int_{V} u^{0}(z) v_{i} \theta^{* j}(y, v) \psi(0, y, v) \psi^{*}(0, y, v) \frac{\partial^{2} \phi}{\partial z_{i} \partial z_{j}} \mathrm{~d} z \mathrm{~d} y \mathrm{~d} v \\
& +\int_{\mathbb{R}^{N}} \int_{Y} \int_{Y} u^{0}(z) v \cdot \nabla_{x} \psi(0, y, v) \psi^{*}(0, y, v) \phi(z) \mathrm{d} z \mathrm{~d} y \mathrm{~d} v \\
& +\int_{\mathbb{P}^{N}} \int_{Y} \int_{Y} \int_{V}\left(\lambda_{i j}^{2} z_{i} z_{j}+\eta\right) f\left(0, y, v^{\prime}, v\right) \psi\left(0, y, v^{\prime}\right) \psi^{*}(0, y, v) u^{0}(z) \phi(z) \mathrm{d} z \mathrm{~d} y \mathrm{~d} v \\
& +\int_{\mathbb{R}^{N}} \int_{Y} \int_{V}\left(\operatorname{div}\left(\bar{B}^{*} z u^{0}\right)-\operatorname{div}(J)(0) u^{0}\right) \phi^{0} \mathrm{~d} z \mathrm{~d} y \mathrm{~d} v \\
& =\int_{\mathbb{R}^{N}} \int_{Y} \int_{V} \int_{V} f\left(0, y, v^{\prime}, v\right) \psi\left(0, y, v^{\prime}\right) \psi^{*}(0, y, v) \tilde{q}\left(z, v^{\prime}\right) \phi(z) \mathrm{d} z \mathrm{~d} y \mathrm{~d} v .
\end{aligned}
$$

To conclude the proof, we remark that

$$
\int_{Y} \int_{V} v_{i} \theta^{* j} \psi \psi^{*}=-\int_{Y} \int_{V} v_{j} \psi \psi^{*} \theta^{i}=-\int_{Y} \int_{V} v_{j} \psi^{*} \chi^{i}=\bar{D}_{j i}
$$

which we obtain by multiplying (64) by $\theta^{j}$ and integrating by parts. Thus, (66) is nothing but the homogenized equation (63).

\section{Cell problems with Drift}

This section is devoted to the so-called drift or $\theta$-exponential cell problems, which allow us to prove that the asymptotic expansions and the two-scale method yield the same results.

\section{1. $\boldsymbol{\theta}$-exponential cell problems}

Let $\theta$ be a constant vector in $\mathbb{R}^{N}$. We introduce the following $\theta$-exponential cell problem

$$
\left\{\begin{array}{l}
v \cdot \nabla_{y} \psi_{\theta}+\Sigma \psi_{\theta}=\int_{V} \sigma \psi_{\theta} \mathrm{d} v^{\prime}+\lambda^{\infty}(x, \theta) \int_{V} f \psi_{\theta} \mathrm{d} v^{\prime} \\
y \mapsto \psi_{\theta}(x, y, v) \exp (\theta \cdot y) Y-\text { periodic, }
\end{array}\right.
$$

and its adjoint problem

$$
\left\{\begin{array}{l}
-v \cdot \nabla_{y} \psi_{\theta}^{*}+\Sigma \psi_{\theta}^{*}=\int_{V} \sigma^{*} \psi_{\theta}^{*} \mathrm{~d} v^{\prime}+\lambda^{\infty}(x, \theta) \int_{V} f^{*} \psi_{\theta}^{*} \mathrm{~d} v^{\prime} \\
y \mapsto \psi_{\theta}^{*}(x, y, v) \exp (-\theta \cdot y) Y-\text { periodic. }
\end{array}\right.
$$

It is convenient to perform the following change of unknowns $\varphi_{\theta}(x, y, v)=\psi_{\theta}(x, y, v) \exp (\theta \cdot y)$ and $\varphi_{\theta}^{*}(x, y, v)$ $=\psi_{\theta}^{*}(x, y, v) \exp (-\theta \cdot y)$. They solve the following problems

$$
\left\{\begin{array}{l}
v \cdot \nabla_{y} \varphi_{\theta}-v \cdot \theta \varphi_{\theta}+\Sigma \varphi_{\theta}=\int_{V} \sigma \varphi_{\theta} \mathrm{d} v^{\prime}+\lambda^{\infty}(x, \theta) \int_{V} f \varphi_{\theta} \mathrm{d} v^{\prime} \\
y \mapsto \varphi_{\theta}(x, y, v) Y \text { - periodic, }
\end{array}\right.
$$

and

$$
\left\{\begin{array}{l}
-v \cdot \nabla_{y} \varphi_{\theta}^{*}-v \cdot \theta \varphi_{\theta}^{*}+\Sigma \varphi_{\theta}^{*}=\int_{V} \sigma^{*} \varphi_{\theta}^{*} \mathrm{~d} v^{\prime}+\lambda^{\infty}(x, \theta) \int_{V} f^{*} \varphi_{\theta}^{*} \mathrm{~d} v^{\prime} \\
y \mapsto \varphi_{\theta}^{*}(x, y, v) Y \text { - periodic. }
\end{array}\right.
$$


Such problems were studied in [7], where, among other properties, it is proved that, for any $x \in \Omega$, the function $\theta \mapsto \lambda^{\infty}(x, \theta)$ admits a unique critical point $\theta_{0}$ (depending on $x$ ) which is a maximum and that

$$
\nabla_{\theta} \lambda^{\infty}(x, \theta)=J(x, \theta)=\int_{Y} \int_{V} v \psi_{\theta} \psi_{\theta}^{*}
$$

Therefore, $\theta_{0}$ is uniquely characterized by $J\left(x, \theta_{0}\right)=0$. Our previous notation $J(x)$, defined by (9), coincides with $J(x, 0)$ as defined in (71). Our assumption (H4) just means that for $x=x_{0}=0$ we have $\theta_{0}=0$.

\subsection{On a relation between the limit drift $\bar{B}$ and the cell drift $J$}

We are now in position to prove a result announced in Remark 2.2, namely that

$$
\bar{B}_{i j}=\frac{\partial J_{j}}{\partial x_{i}}(x=0)
$$

Deriving (69) with respect to $\theta_{j}$ yields

$$
v \cdot \nabla_{y} \partial_{\theta_{j}} \varphi_{\theta}-v_{j} \varphi_{\theta}-v \cdot \theta \partial_{\theta_{j}} \varphi_{\theta}+\Sigma \partial_{\theta_{j}} \varphi_{\theta}=\int_{V} \sigma \partial_{\theta_{j}} \varphi_{\theta}+\lambda^{\infty}(x, \theta) \int_{V} f \partial_{\theta_{j}} \varphi_{\theta}+\partial_{\theta_{j}} \lambda^{\infty} \int_{V} f \varphi_{\theta} .
$$

Multiplying by $\varphi_{\theta}^{*}$, and integrating on $Y \times V$ yields

$$
-\int_{Y} \int_{V} v_{j} \varphi_{\theta} \varphi_{\theta}^{*}=\partial_{\theta_{j}} \lambda^{\infty} \int_{Y} \int_{V}\left(\int_{V} f \varphi_{\theta}\right) \varphi_{\theta}^{*}
$$

or equivalently

$$
-J(\theta, x)=-\int_{Y} \int_{V} v \psi_{\theta} \psi_{\theta}^{*}=\nabla_{\theta} \lambda^{\infty} \int_{Y} \int_{V}\left(\int_{V} f \psi_{\theta}\right) \psi_{\theta}^{*}
$$

Deriving (72) with respect to $x_{i}$, we obtain

$$
\begin{gathered}
v \cdot \nabla_{y} \partial_{x_{i} \theta_{j}}^{2} \varphi_{\theta}-v_{j} \partial_{x_{i}} \varphi_{\theta}-v \cdot \theta \partial_{x_{i} \theta_{j}}^{2} \varphi_{\theta}+\Sigma \partial_{x_{i} \theta_{j}}^{2} \varphi_{\theta}+\partial_{x_{i}} \Sigma \partial_{\theta_{j}} \varphi_{\theta}=\int_{V}\left(\sigma \partial_{x_{i} \theta_{j}}^{2} \varphi_{\theta}+\partial_{x_{i}} \sigma \partial_{\theta_{j}} \varphi_{\theta}\right) \\
+\lambda^{\infty} \int_{V}\left(f \partial_{x_{i} \theta_{j}}^{2} \varphi_{\theta}+\partial_{x_{i}} f \partial_{\theta_{j}} \varphi_{\theta}\right)+\partial_{x_{i}} \lambda^{\infty} \int_{V} f \partial_{\theta_{j}} \varphi_{\theta}+\partial_{\theta_{j}} \lambda^{\infty} \int_{V}\left(f \partial_{x_{i}} \varphi_{\theta}+\partial_{x_{i}} f \partial_{\theta_{j}} \varphi_{\theta}\right) \\
+\partial_{x_{i} \theta_{j}}^{2} \lambda^{\infty} \int_{V} f \varphi_{\theta}
\end{gathered}
$$

We write this equation at $(x, \theta)=\left(x_{0}, \theta_{0}\right)=(0,0)$. Therefore, assumptions (H3) and (H4) imply that the terms $\partial_{x_{i}} \lambda^{\infty}$ and $\partial_{\theta_{j}} \lambda^{\infty}$ vanish. Multiplying by $\varphi_{\theta}^{*}$ and integrating on $Y \times V$ yields at $x=0$

$$
\begin{aligned}
\int_{Y} \int_{V}-v_{j} \partial_{x_{i}} \varphi_{0} \varphi_{0}^{*}+\Sigma_{i}^{1} \partial_{\theta_{j}} \varphi_{0} \varphi_{0}^{*}= & \int_{Y} \int_{V}\left(\int_{V} \sigma_{i}^{1} \partial_{\theta_{j}} \varphi_{0}\right) \varphi_{0}^{*}+\lambda^{\infty}\left(\int_{V} f_{i}^{1} \partial_{\theta_{j}} \varphi_{0}\right) \varphi_{0}^{*} \\
& +\partial_{x_{i} \theta_{j}}^{2} \lambda^{\infty} \int_{Y} \int_{V} \int_{V} f \varphi_{0} \varphi_{0}^{*}
\end{aligned}
$$

Remember our normalization for the eigenvectors

$$
\int_{Y} \int_{V}\left(\psi_{\theta}\right)^{2} \mathrm{~d} y \mathrm{~d} v=1 \text { and } \int_{Y} \int_{V}\left(\int_{V} f \psi_{\theta}\right) \psi_{\theta}^{*} \mathrm{~d} y \mathrm{~d} v=1 .
$$


With this convention, deriving (73) with respect to $x_{i}$ yields

$$
-\frac{\partial J_{j}}{\partial x_{i}}=\frac{\partial^{2} \lambda^{\infty}}{\partial x_{i} \partial \theta_{j}}
$$

At $(x, \theta)=(0,0)$, we have obviously $\partial_{x_{i}} \varphi_{\theta}=\psi_{i}^{1}$ by comparing (19) and equations (69). Similarly, comparing (22) and (72), we have $\partial_{\theta_{j}} \varphi_{\theta}=-\chi^{j}$. And thus, equations (74) and (75) yield

$$
\bar{B}_{i j}=\int_{Y} \int_{V}\left(v_{j} \psi_{i}^{1}+\Sigma_{i}^{1} \chi^{j}-\int_{V} \sigma_{i}^{1} \chi^{j}-\lambda^{\infty} \int_{V} f_{i}^{1} \chi^{j}\right) \psi^{0 *}=\partial_{x_{i}} J_{j}
$$

which is the desired result.

Remark 6.1. If we assumed, instead of (H4), the much stronger assumption that the drift flux $J(x)$ vanishes in a neighborhood of $x=0$, it would be possible to prove in a much simpler way (avoiding $\theta$-exponential cell problems) that $\bar{B}=\nabla_{x} J(0)=0$. Indeed, the following equation, similar to (22) is solvable in a neighborhood of $x=0$,

$$
v \cdot \nabla \chi^{j}+\Sigma \chi^{j}=\int_{V} \sigma \chi^{j}+\lambda^{\infty}(x) \int_{V} f \chi^{j}-v_{j} \psi
$$

Thus, differentiating this equation with respect to $x_{i}$ at $x=0$ yields

$$
v \cdot \nabla \partial_{x_{i}} \chi^{j}+\Sigma \partial_{x_{i}} \chi^{j}=\int_{V} \sigma \partial_{x_{i}} \chi^{j}+\lambda^{\infty} \int_{V} f \partial_{x_{i}} \chi^{j}-\Sigma_{i}^{1} \chi^{j}+\int_{V} \sigma_{i}^{1} \chi^{j}+\lambda^{\infty} \int_{V} f_{i}^{1} \chi^{j}-v_{j} \psi_{i}^{1},
$$

which is also solvable by definition. Therefore, the solvability condition of this last equation is satisfied, and this precisely means that $\bar{B}_{i j}=0$.

\subsection{On a generalization of the convergence result}

In view of the properties of $\lambda^{\infty}(x, \theta)$, it is natural to replace our hypotheses (H3) and (H4) by a new one, (H5), which states that there exists a unique couple $\left(x_{0}, \theta_{0}\right) \in \Omega \times \mathbb{R}^{N}$, such that

$$
\begin{aligned}
\theta \longmapsto & \lambda^{\infty}\left(x_{0}, \theta\right) \text { reaches its maximum at } \theta=\theta_{0} \\
\text { (H5) } \quad x \longmapsto & \lambda^{\infty}\left(x, \theta_{0}\right) \text { reaches its minimum at } x=x_{0} \\
& \text { with } \nabla_{x} \nabla_{x} \lambda^{\infty}\left(x_{0}, \theta_{0}\right) \text { positive definite. }
\end{aligned}
$$

Notice that (H3) and (H4) are indeed equivalent to (H5) when $\left(x_{0}, \theta_{0}\right)=(0,0)$.

We now explain a new phenomenon occurring when hypothesis (H3) and (H4) are not satisfied, but are replaced by (H5). Of course, we still need the hypotheses (H1, H2). Instead of writing the first eigenfunction $\phi^{\varepsilon}$ of (1) in the form

$$
\phi^{\varepsilon}(x, v)=\psi\left(x, \frac{x}{\varepsilon}, v\right) u^{\varepsilon}(x, v),
$$

we introduce a new factorization and write

$$
\phi^{\varepsilon}(x, v)=\psi_{\theta_{0}}\left(x, \frac{x}{\varepsilon}, v\right) u_{\theta_{0}}^{\varepsilon}(x, v),
$$

where $\psi_{\theta_{0}}$ is the solution of $(67)$. At $\left(x_{0}, \theta_{0}\right)$, by definition we have $J\left(x_{0}, \theta_{0}\right)=0$, and thus our whole study is still valid with this new factorization principle. Remark that (H4) was of crucial importance in the previous 
section because it was a Fredholm solvability condition, but it is now replaced by $J\left(x_{0}, \theta_{0}\right)=0$ which is a consequence of the first assumption in (H5). Therefore, we can prove that

$$
u_{\theta_{0}}^{\varepsilon}(x, v) \approx \exp \left(-\frac{M\left(\theta_{0}\right)}{2 \varepsilon}\left(x-x_{0}\right) \cdot\left(x-x_{0}\right) .\right.
$$

We skip the details for the sake of brevity. Formally, this indicates that, in the limit $\varepsilon \rightarrow 0$, the asymptotic behavior of $\phi^{\varepsilon}$ is changed and we have

$$
\phi^{\varepsilon}(x, v) \approx \varphi_{\theta_{0}}\left(x_{0}, \frac{x}{\varepsilon}, v\right) \exp \left(-\theta_{0} \cdot \frac{x-x_{0}}{\varepsilon}\right) \exp \left(-\frac{M\left(\theta_{0}\right)}{2 \varepsilon}\left(x-x_{0}\right) \cdot\left(x-x_{0}\right),\right.
$$

where $\varphi_{\theta_{0}}$ is periodic. Note that the approximation sign in (77) is purely formal and has no real justification since we can not pass easily from $(76)$ to $(77)$ by multiplying by a function, $\exp \left(-\theta_{0} \cdot\left(x-x_{0}\right) / \varepsilon\right)$, which is widely unbounded as $\varepsilon$ goes to zero. At least, $(77)$ is an indication that there is a competition between the concentration term and the drift term and it seems to induce a new concentration point for $\phi^{\varepsilon}$. Formally, (77) suggests that this new concentration point $x_{1}$ is given by

$$
x_{1}=x_{0}+M\left(\theta_{0}\right)^{-1} \theta_{0},
$$

but a more detailed analysis is required to find the precise value of $x_{1}$. Remark also that it is not clear how to check assumption (H5) on specific examples of coefficients.

\section{REFERENCES}

[1] G. Allaire, Homogenization and two scale convergence. SIAM 23 (1992) 1482-1518.

[2] G. Allaire and G. Bal, Homogenization of the critically spectral equation in neutron transport. ESAIM: M2AN 33 (1999) 721-746.

[3] G. Allaire and Y. Capdeboscq, Homogenization of a spectral problem in neutronic multigroup diffusion. Comput. Methods Appl. Mech. Engrg. 187 (2000) 91-117.

[4] G. Allaire and A. Piatnitski, Uniform spectral asymptotics for singularly perturbed locally periodic operators. Com. Partial Differential Equations 27 (2002) 705-725.

[5] P. Anselone, Collectively compact operator approximation theory. Prentice-Hall, Englewood Cliffs, NJ (1971).

[6] G. Bal, Couplage d'équations et homogénéisation en transport neutronique, Ph.D. Thesis. Paris 6 (1997).

[7] - Homogenization of a spectral equation with drift in linear transport. ESAIM: COCV 6 (2001) 613-627.

[8] A. Bensoussan, J.-L. Lions and G. Papanicolaou, Boundary layer and homogenization of transport processes. Publ. RIMS Kyoto Univ. (1979) 53-157.

[9] Y. Capdeboscq, Homogénéisation des modèles de diffusion en neutronique, Ph.D. Thesis. Paris 6 (1999).

[10] F. Chatelin, Spectral approximation of linear operators. Academic Press (1983).

[11] R. Dautray and J.-L. Lions, Mathematical analysis and numerical methods for science and technology. Springer Verlag, Berlin (1993).

[12] P. Degond, T. Goudon and F. Poupaud, Diffusion limit for nonhomogeneous and non-micro-reversible processes. Indiana Univ. Math. J. 49 (2000) 1175-1198.

[13] J. Glimm and A. Jaffe, Quantum Physics. A Functional Integral Point of View. Springer-Verlag, New York, Berlin (1981).

[14] F. Golse, P.L. Lions, B. Perthame and R. Sentis, Regularity of the moments of the solution of a transport equation. J. Funct. Anal. 76 (1988) 110-125.

[15] F. Golse, B. Perthame and R. Sentis, Un résultat de compacité pour les équations de transport et application au calcul de la limite de la valeur propre principale d'un opérateur de transport. C. R. Acad. Sci. Paris (1985) 341-344.

[16] T. Goudon and A. Mellet, Diffusion approximation in heterogeneous media. Asymptot. Anal. (to appear).

[17] T. Goudon and F. Poupaud, Approximation by homogenization and diffusion of kinetic equations. Comm. Partial Differential Equations 26 (2001) 537-569.

[18] S. Kozlov, Reductibility of quasiperiodic differential operators and averaging. Transc. Moscow Math. Soc. 2 (1984) 101-126.

[19] E. Larsen, Neutron transport and diffusion in inhomogeneous media (1). J. Math. Phys. (1975) 1421-1427.

$[20]$ - Neutron transport and diffusion in inhomogeneous media (2). Nuclear Sci. Engrg. (1976) 357-368.

[21] E. Larsen and J. Keller, Asymptotic solution of neutron transport problems for small mean free paths. J. Math. Phys. (1974) $75-81$. 
[22] M. Mokhtar-Kharoubi, Les équations de la neutronique, Thèse de Doctorat d'État. Paris XIII (1987).

[23] M. Mokhtar-Kharoubi, Mathematical topics in neutron transport theory. World Scientific Publishing Co. Inc., River Edge, NJ (1997).

[24] A. Piatnitski, Asymptotic behaviour of the ground state of singularly perturbed elliptic equations. Commun. Math. Phys. 197 (1998) 527-551.

[25] J.E. Potter, Matrix quadratic solutions, J. SIAM Appl. Math. 14 (1966) 496-501.

[26] D.L. Russel, Mathematics of finite-dimensional control systems, theory and design. Lecture Notes in Pure Appl. Math. 43 (1979).

[27] R. Sentis, Study of the corrector of the eigenvalue of a transport operator. SIAM J. Math. Anal. (1985) 151-166. 